\title{
Extensive T-cell epitope repertoire sharing among human proteome, gastrointestinal microbiome, and pathogenic bacteria: implications for the definition of self
}

\author{
Robert D. Bremel* and E. Jane Homan \\ EigenBio LLC, Madison, WI, USA
}

OPEN ACCESS

Edited by: Nick Gascoigne,

National University of Singapore,

Singapore

Reviewed by:

Paula Kavathas,

Yale University School of Medicine,

Tomasz Zal,

University of Texas MD Anderson Cancer Center, USA

*Correspondence: Robert D. Bremel robert_breme/@eigenbio.com

Specialty section: This article was submitted to T Cell

Biology, a section of the journal Frontiers in Immunology

Received: 16 July 2015 Accepted: 08 October 2015 Published: 22 October 2015

Citation:

Bremel RD and Homan EJ (2015) Extensive T-cell epitope repertoire sharing among human proteome, gastrointestinal microbiome, and pathogenic bacteria: implications for the definition of self.

Front. Immunol. 6:538. doi: 10.3389/fimmu.2015.00538
T-cell receptor binding to $\mathrm{MHC}$-bound peptides plays a key role in discrimination between self and non-self. Only a subset, typically a pentamer, of amino acids in a MHC-bound peptide form the motif exposed to the T-cell receptor. We categorize and compare the T-cell exposed amino acid motif repertoire of the total proteomes of two groups of bacteria, comprising pathogens and gastrointestinal microbiome organisms, with the human proteome and immunoglobulins. Given the maximum $20^{5}$, or 3.2 million of such motifs that bind T-cell receptors, there is considerable overlap in motif usage. We show that the human proteome, exclusive of immunoglobulins, only comprises three quarters of the possible motifs, of which $65.3 \%$ are also present in both composite bacterial proteomes. Very few motifs are unique to the human proteome. Immunoglobulin variable regions carry a broad diversity of T-cell exposed motifs (TCEMs) that provides a stratified random sample of the motifs found in pathogens, microbiome, and the human proteome. Individual bacterial genera and species vary in the content of immunoglobulin and human proteome matched motifs that they carry. Mycobacteria and Burkholderia spp carry a particularly high content of such matched motifs. Some bacteria retain a unique motif signature and motif sharing pattern with the human proteome. The implication is that distinguishing self from non-self does not depend on individual TCEMs, but on a complex and dynamic overlay of signals wherein the same TCEM may play different roles in different organisms, and the frequency with which a particular TCEM appears influences its effect. The patterns observed provide clues to bacterial immune evasion and to strategies for intervention, including vaccine design. The breadth and distinct frequency patterns of the immunoglobulin-derived peptides suggest a role of immunoglobulins in maintaining a broadly responsive T-cell repertoire.

Keywords: T-cell repertoire, human proteome, microbiome, T-cell epitope, immunogenetics, central tolerance, Mycobacterium, Burkholderia 


\section{INTRODUCTION}

A central theme of immunology is the ability of the adaptive immune system to differentiate self from non-self. Much of the ability to mount a specific immune response is dependent on T-cell recognition of cognate peptide epitopes in the context of the MHC molecules determined by the host's genetics. In this analysis, we examine the limitations of the T-cell epitope repertoire in distinguishing self from non-self by categorizing and comparing the TCEM repertoire of two groups of bacterial proteomes with that of the human proteome and immunoglobulins.

We have previously reported on the characteristics of T-cell exposed amino acid motifs of peptides bound in MHC molecules in mediating T-cell recognition and polyspecificity (1). T-cell exposed motifs (TCEMs) are the combinations of amino acids, typically pentamers, whose side chains are exposed to T-cell receptors when an epitope peptide is bound within a MHC groove. A TCEM is exposed to the T-cell within the context of the surrounding MHC histotope. Conversely, some of the amino acid side chains of the MHC-bound peptide are hidden to the $\mathrm{T}$-cell receptor and are directed into the MHC groove, where they determine the binding affinity of the peptide to the $\mathrm{MHC}$ and thus the kinetic stability, or dwell time, during which T-cell receptor engagement occurs. Based on the structural analysis of Rudolph et al., three pentameric contact patterns of TCEMs are recognized (2). For MHC I, these comprise amino acids 4,5,6,7,8 of a core 9-mer, and for MHC II two patterns, which we call TCEM IIa and IIb, comprising the amino acids 2,3,5,7,8 and -1,3,5,7,8 of the central 9-mer core of a 15 -mer $(1,2)$. These contact patterns are summarized in Figure 1. A pentamer contact pattern sets the maximum number of TCEM configurations that T-cells recognize at $20^{5}$, or 3.2 million, for each of the contact patterns. This determines the need for polyspecificity, and enables a finite T-cell population to respond to all potential epitopes $(3,4)$.

T-cell exposed motifs provide a framework to understand large protein sets from the perspective of T-cell recognition. We showed previously that in $\operatorname{IgV}$ the amino acids making up the TCEM assort independently of the amino acids

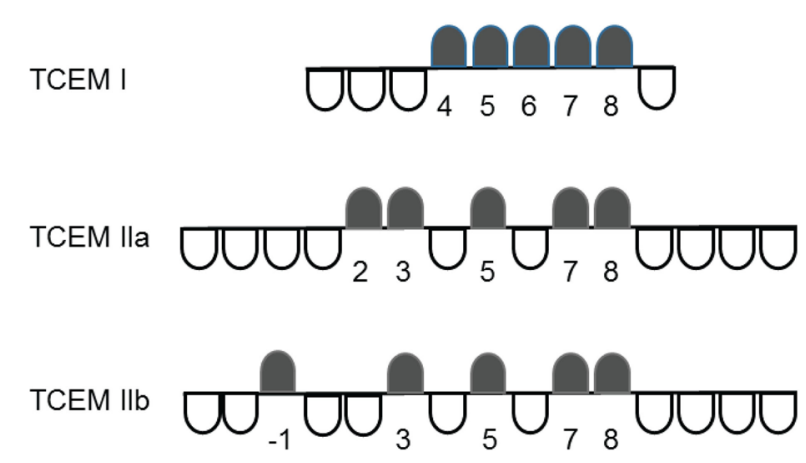

FIGURE 1 | T-cell-exposed motif patterns. In each contact pattern the black markers indicate the T-cell exposed amino acids, while the white markers indicate the groove exposed amino acids. The numbers identify amino acids positions in each motif using the $\mathrm{MHC}$ groove pocket numbering scheme. making up the intercalated groove exposed motif (GEM) (1). The T-cell engagement is with the histotope and the exposed amino acids of the MHC-bound peptide, but not with the groove exposed amino acids. The T-cell-TCEM interaction is not influenced by the GEM except in so far as the latter determines the binding affinity and thus how long the peptide remains associated with the MHC to enable repeated T-cell interactions to occur. Therefore, we approached this analysis by looking first at the TCEM motifs alone.

For any protein, there is a gradation in frequency of T-cell encounter with different TCEMs present in that protein. Common TCEMs will cause repeated clonal stimulation leading to a buildup of a larger population of cognate T-cells, whereas rare TCEM will have fewer encounters and generate a smaller cognate population (4). The motif usage patterns previously observed in the $\mathrm{IgV}$ prompted us to examine the patterns of occurrence of TCEMs in other large protein sets relevant to the function of the immune system: the human proteome exclusive of the immunoglobulins, and two exemplary groups of bacteria. The first group of bacteria comprises 67 species in 35 genera from the gastrointestinal microbiome. The second group comprises up to five proteomes each of 28 species in 12 genera of pathogenic bacteria. We characterized the patterns of potential TCEM present within each proteome dataset, and compared them to each other, and to the repertoire of motifs in $\mathrm{IgV}$ regions.

The interdependency of the B-cell immunoglobulin variable regions (IgV) and T-cell suppressor repertoires has long been recognized (5). Peptides derived from $\mathrm{IgV}$ are processed, bound in MHC, and presented to T-cells as are other peptides of other origins (6-8). B-cells present their own endogenous IgV-derived peptides in MHC (9), a process that is thought to play a key role in determining T-cell diversity $(10,11)$. We reported that within $\mathrm{IgV}$ regions there is a high rate of repetitive appearance of TCEM of both germline-origin and those resulting from somatic hypermutation (1). In a sample of "normal" IgV, from which sequences originating from immunopathologies had been eliminated, estimated to be approximately equivalent to $1 \%$ of the population of B-cell clonotypes in any one individual (12), we showed that approximately $50 \%$ of the TCEMs recur in at least 1 in every 1024 B-cell clonal products. We proposed that the peptides derived from IgV, and presented on MHC by B-cells and by other antigen presenting cells (APCs), provide the constantly cycling peptide diversity needed to maintain an active T-cell repertoire required to mount an appropriate immune response to a vast diversity of antigens, both self and non-self, of pathogen or environmental origin.

We find that the human proteome uses only about three quarters of the total possible pentamers in each TCEM contact pattern and thus the immune system has evolved mechanisms to recognize and respond appropriately to the other quarter of the motifs. The bacterial species share motifs with the human proteome but in addition contain nearly all of the TCEMs not found in the human proteome, a characteristic that highlights the importance in immune recognition of the sampling of the microbiome by the gut associated lymphatic tissue. The IgV-derived TCEMs provide a broad representation of the other proteomes and also generate TCEMs not found in any of the three proteome 
datasets. Within the bacterial pathogen dataset we can differentiate protein-specific, genus-specific, and some species specific patterns in TCEM usage. Overall, the relationship points to the role of TCEM frequency in pathogen immune evasion, tolerance of commensals, and the potential for generation of autoimmune responses following bacterial infection or disturbance of the microbiome.

\section{MATERIALS AND METHODS}

\section{Protein Sets}

Four groups of protein sequences were assembled:

a. Human proteome (human proteome): this group comprises 88,146 proteins, including multiple isoforms of proteins, with an average length of 384 amino acids. As a whole, the set thus provides about a fourfold redundancy of the approximately 20,000 proteins in any individual human proteome. This set of proteins was assembled from the UniProt repository ${ }^{1}$ (13), and based on the UniProt annotations it was manually curated to remove the proteins containing immunoglobulin variable regions. The final curated set comprised $3.3 \times 10^{7}$ total TCEM in each of the three contact patterns.

b. Gastrointestinal microbiome bacteria (microbiome): the proteomes were assembled from the NIH Human Microbiome Project Reference Genomes database ${ }^{2}$ and included 67 species in 35 genera (listed in Table S1 in Supplementary Material). This set comprised a total of 378,061 proteins with an average length of 290 amino acids. This is not a set of fully annotated proteomes and may include some partial proteomes. The final set comprised $1.09 \times 10^{8}$ total TCEM in each of the three contact patterns and is thus of a comparable total size to the bacterial pathogen set in (c).

c. Exemplary bacterial pathogens (pathogens) comprising 28 species each represented by up to five proteomes (132 in all) selected at random from the complete proteomes of each genus available at PATRIC ${ }^{3}$ (14). The genera included were: Bordetella, Brucella, Burkholderia, Chlamydia, Clostridium, Coxiella, Francisella, Mycobacterium, Neisseria, Staphylococcus, Streptococcus, and Ureaplasma. Species and strains are shown in Table S1 in Supplementary material. Proteomes ranged in size from approximately 6000 proteins (Burkholderia) to 689 (Ureaplasma). In total 427,906 proteins were included in the final pathogen dataset, comprising $1.16 \times 10^{8}$ total TCEM in each of the three TCEM contact patterns.

d. Immunoglobulin variable regions ( $\operatorname{IgV})$ : the 40,000 heavy chain $\mathrm{V}$ regions were assembled as previously described (1). A further 16,000 light chain variable regions were also retrieved from Genbank and curated to remove those derived from immunopathologies, using the same criteria as described for the heavy chains. The final reference databases

${ }^{1}$ www.uniprot.org

${ }^{2}$ www.hmpdacc.org/HMRGD

${ }^{3}$ www.patricbrc.org comprised approximately $6.4 \times 10^{6}$ total TCEM, including 325,000 unique motifs in each of the three contact patterns.

\section{Analysis of T-Cell Exposed Motifs}

To construct the TCEM sets, concatenated amino acid FASTA files of each of the proteomes were assembled, from which each protein in the proteome was then decomposed into sets of 9-mer and 15-mer peptides, each offset by a single amino acid and placed in successive rows of a JMP ${ }^{\circledR}$ data table. Three different TCEM contact patterns were constructed from each peptide in corresponding columns using column formulas of $\mathrm{JMP}^{\circledR}$. These were TCEM I comprising amino acids $(4,5,6,7,8)$ of a core MHC-I 9-mer, and TCEM IIa and TCEM IIb comprising amino acids corresponding to amino acids $(2,3,5,7,8)$ and $(-1,3,5,7,8)$ of the central 9-mer core of a 15-mer $(1,2)$. Hence, each 9-mer yielded a motif in the format $\sim \sim \sim \mathrm{XXXXX} \sim$ where $\sim$ represents a non-exposed amino acid, and each 15-mer yielded a motif in the format $\sim \sim \sim \sim \mathrm{XX} \sim \mathrm{X} \sim \mathrm{XX} \sim \sim \sim \sim$ or $\sim \sim \mathrm{X} \sim \sim \mathrm{X} \sim \mathrm{X} \sim \mathrm{XX} \sim \sim \sim \sim$. The GEM responsible for the binding interactions was similarly assembled as the intercalated set of non-exposed amino acids in each peptide. The unique identifier of each protein, the peptide, and the $\mathrm{N}$ terminal positions of each peptide in the parent protein within the proteome were retained with each TCEM. In this way TCEM sets were hyperlinked to the parental proteins by JMP ${ }^{\circledR}$ so that the parental proteins could be readily retrieved for other analyses. Three sets of tables, one for each TCEM contact pattern, were constructed for each protein set.

\section{Frequency and Diversity Analysis of Proteome TCEM}

Preliminary analysis of TCEM frequencies in the different proteomes indicated that they all exhibited a log-normal distribution pattern characterized by two parameters: a mean $(\mu)$ and a scale factor $(\sigma)$ analogous to an SD. In keeping with our previous use of a $\log 2$ base for characterizing the frequency-of-occurrence of TCEM in IgV (1), each motif was characterized according to the frequency of occurrence in each particular proteome. The diversity of TCEM occurrence in each proteome was computed as the Shannon Entropy $\mathrm{H}$ as

$$
H=-\sum_{1}^{\mathrm{N}} \mathrm{p} \log 2 \mathrm{p}
$$

where $p=$ frequency of occurrence of a particular TCEM and $N=$ total number of TCEM in the proteome for the contact pattern.

\section{TreeMapping of Multivariate Datasets}

Treemaps were used to visualize underlying patterns in large multivariate datasets and were particularly useful for displaying hierarchical data as a set of nested rectangles. Briefly, TCEM usage in each proteome was tallied for each of the proteomes. Each of the proteomes exhibited a pattern of motif use, some of which was shared with other organisms and the human proteome, and some of which was unique to the particular organism. An $N \times M$ 
matrix where $N=3.2$ million and $M=$ the number of proteins was created which contained the tallies of the number of each of the particular TCEM. Then a binary string was created which described the presence or absence of each of the motifs across all proteomes. This string pattern was used to create a treemap of comparative TCEM content across proteomes. This process works well for a number of proteomes within a genus, and across genera and provides a useful way to compare and visualize large proteomic datasets.

\section{Immunoglobulin TCEM Frequencies}

The TCEM in each of the proteomes described were characterized as to whether or not they were present in immunoglobulins and, if present, their particular frequency of occurrence in immunoglobulins. It should be noted that in this paper we use the frequency of TCEM occurrence based on total TCEM within the whole immunoglobulin dataset. This differs from our prior analysis where only IgV data was analyzed (1), and hence where frequencies were standardized to the number of $\operatorname{IgV}$ clonotypes.

\section{Software}

All data processing, pattern analysis and statistical analysis were done with JMP ${ }^{\circledR}$ v12 from SAS Institute (Cary, NC, USA).

\section{RESULTS}

All data described in the Section "Results" are for TCEM IIa, corresponding to MHC II mediated CD4+ responses. The corresponding results of analysis for TCEM I, for MHC I CD8+ responses, is appended in the Supplemental Tables and Figures. Results for TCEM IIb are not shown but are very closely analogous to TCEM IIa, as are results observed for all three contact patterns.

\section{TCEM Content and Frequency Distribution in Human Proteome, Microbiome, and Pathogen Datasets}

Based on the pentamer contact pattern, there is a maximum of $20^{5}$ possible TCEMs ( 3.2 million) for each of the three contact patterns TCEM I, IIa and IIb. Table 1 shows the frequency distribution of TCEM IIa in each proteomic dataset. None of the proteome datasets uses the full complement of possible TCEMs and there is a varying degree of overlap between proteome datasets. The Human proteome dataset comprises 2,412,699 unique

\begin{tabular}{rcccc}
\hline \multicolumn{5}{l}{ TABLE 1 Composition of TCEM lla proteome datasets. } \\
\hline TCEM count & Human & Pathogen & Microbiome & $\begin{array}{c}\text { Percent of } \\
\mathbf{3 . 2} \text { million }\end{array}$ \\
\hline $2,090,606$ & 1 & 1 & 1 & 65.3 \\
216,262 & 1 & 0 & 1 & 6.9 \\
413,177 & 0 & 1 & 1 & 12.9 \\
201,400 & 0 & 0 & 1 & 6.3 \\
46,575 & 1 & 1 & 0 & 1.5 \\
59,256 & 1 & 0 & 0 & 1.9 \\
51,041 & 0 & 1 & 0 & 1.6 \\
121,683 & 0 & 0 & 0 & 3.8
\end{tabular}

TCEM, or only about three quarters of the maximum possible motifs (Table 2; Figure 2). Of these, 2,090,606 are shared with both the Microbiome and Pathogen composite proteome sets. The Human proteome shares a further 216,262 motifs with only the Microbiome. A smaller set of 46,575 additional TCEMs is shared by only the Human proteome and the Pathogens. The Pathogen set and the Microbiome sets share 413,177 motifs that are missing from the Human proteome, however this reflects a more complex underlying species-specific relationship (Tables S6 and S7 in Supplementary material). Only 59,256 motifs are completely unique to the Human proteome and not found in the bacterial sets. A final 121,683 possible motifs are not used by any of the proteome sets, as discussed further below.

The frequency distribution of motif usage within each of the three proteome sets is log-normal (KSL goodness of fit $p<0.001$ (15). As is shown in Figure 3A both the mean $(\mu)$ frequency and the variation $(\sigma)$ in occurrence are similar between the three different proteomic data sets. The $\log 2$ mean of the distributions is about $\mu=-20$ ( $\mu=-20.3$ for human proteome) for each of the proteomes $\left(1 / 2^{20}=1 / 1,048,576\right)$ or a somewhat greater frequency than $1 / 3,200,000$ that would be expected for a totally random distribution. Moreover, a very slight skewing of the histograms can be seen relative to the overlayed normal distribution curve. The crosshatched insets are the IgV TCEM frequency distribution, which also show a log-normal distribution. The size of the $\operatorname{IgV}$ database used in this analysis is approximately $1 \%$ of the total clonotypes that might be found in a single human (12), but even this relatively small sample of total clonotypes provides a considerable intersection with the proteomic data (Table 2). Additionally, allowing for a single conservative amino acid replacement (16, 17) at any of the five amino acid positions in the TCEM pentamer expands coverage by the IgV-derived motifs to about half of the total motifs in the proteomes (Figure 3B). Comparison of the distribution means in Figures 3A,C shows that in each case the mean of the $\operatorname{IgV}$ distribution is slightly shifted toward a higher TCEM frequency as compared to the mean of the proteome as a whole ( $\mu$ difference $0.4-0.5 \log 2$ ).

\section{Motif Usage in Immunoglobulin Variable Regions}

The log-normal pattern of TCEM recurrence frequency seen in the three proteome data sets, described above, is very different from the frequency of occurrence pattern of motifs found in the $\mathrm{IgV}$, and as previously reported (1). The $\mathrm{IgV}$ dataset comprises only 322,058 unique motifs in the TCEM IIa contact pattern but these have a distinctive frequency distribution pattern of motif re-use. The overall frequency of germline-origin and somatic hypermutation-origin sequences is seen in Figure 4.

The different underlying basis of the diversity in the $\mathrm{IgV}$, the Human proteome and the two bacterial proteomes is evident in the Shannon entropy of the TCEM motifs in the different datasets (Table 3). Since the frequency of occurrence of TCEM is the base of the metric, the entropy calculation is standardized for dataset size, so the values derive from the differences between the combinatorial frequency patterns of the $\mathrm{IgV}$ and the proteomes. The difference in patterns as well as the differences 
TABLE 2 | Fractional composition of proteomic datasets and fractional matches of IgV TCEM Ila motifs.

\begin{tabular}{|c|c|c|c|c|c|}
\hline Group & Count & Percent of 3.2 million & IgV coverage & IgV motifs & Percent of total IgV motifs \\
\hline Human & 2,412,699 & 75.4 & 11.6 & 279,108 & 85.9 \\
\hline Human excluded ${ }^{\mathrm{b}}$ & 787,301 & 24.6 & 5.5 & 42,950 & 13.3 \\
\hline Pathogen & $2,601,399$ & 81.3 & 11.5 & 298,764 & 92.8 \\
\hline Pathogen excluded ${ }^{b}$ & 598,601 & 18.7 & 3.9 & 23,294 & 7.2 \\
\hline Microbiome & $2,921,445$ & 91.3 & 10.7 & 313,197 & 96.4 \\
\hline Microbiome excluded ${ }^{b}$ & 278,555 & 8.7 & 3.2 & 8,861 & 2.7 \\
\hline Not found in human, pathogen or microbiome ${ }^{a}$ & 121,683 & 3.8 & 2.4 & 2,900 & 0.9 \\
\hline
\end{tabular}

aThis set is included in the three excluded sets marked "b".

${ }^{b}$ Excluded sets.

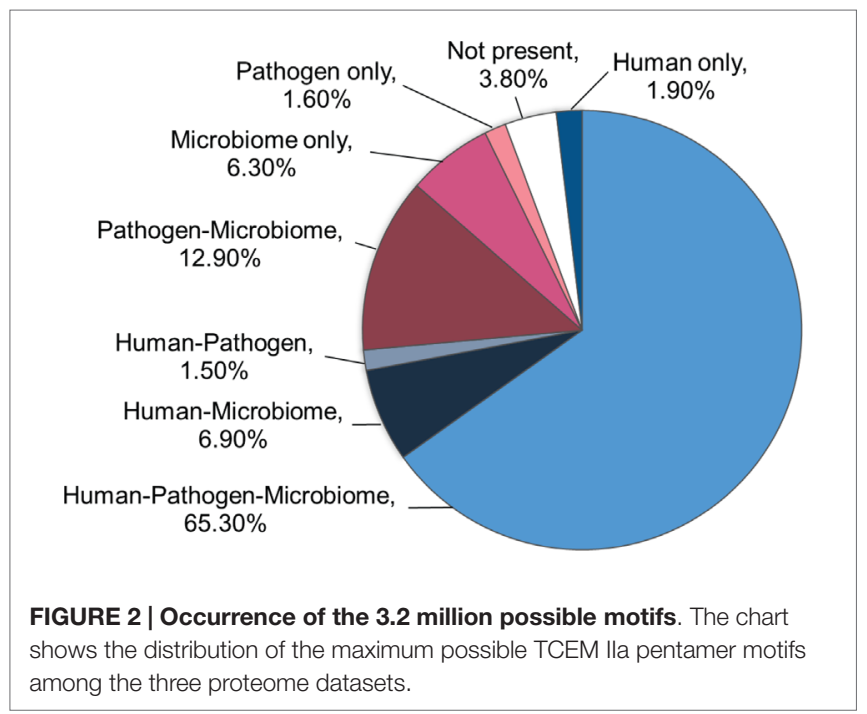

in entropy can be understood by the fact that the $\operatorname{IgV}$ begins as the same germline set (albeit from a number of different gene families) which becomes increasingly diverse during the course of somatic hypermutation and thus comprises highly diverse sequences but which are systematically created from a constant starting point.

Given the differences in overlap between proteome sets, we were interested to examine what the degree of overlap with TCEM used in $\mathrm{IgV}$ was for each proteome. At a full dataset scale there was a slight bias in the IgV matches toward the more commonly encountered motifs (Figure 3A). We compared the frequency of usage of TCEMs in each of the three proteome data sets to that in the $\mathrm{IgV}$ dataset by subsetting the proteomes into the discreet $\mathrm{IgV}$ $\log 2$ based frequency categories (FC) as previously described (1). In this system TCEM motifs are characterized by the number of B-cell clonotypes that display the particular motif; hence a motif designated as FC3 occurs in 1 in 8 B-cell clonotypes and a motif of FC10 occurs in 1 in 1024.

We found that every FC subset of IgV TCEM matched a random sub-sample of each of the three of the proteomic datasets (Figure S3 in Supplementary Material). There is a slight tendency toward the high frequency FC motifs from IgV having greater overlap with the higher frequency motifs in the Human proteome ( $\mu=-18.2$ for FC7 vs. $\mu=-19.7$ for FC12). Overall, however, there is a substantial overlap with the full gamut of different frequency peptides in the three proteome sets. The $\operatorname{IgV}$ frequencies thus effectively comprise a stratified random sample of TCEMs that the immune system will encounter. Motifs from the three proteomes are randomly distributed within each of the IgV FC categories.

That there is a slight bias in the coverage of $\operatorname{IgV}$ can be seen in a different way in Table 2; the IgV TCEM database has a total of 279,108 matches of the 2,412,699 motifs in the Human proteome, an $11.6 \%$ match. This match is with $85 \%$ of the $\mathrm{IgV}$ database, which in total approximates only $1 \%$ of total B-cell clonotypes that an individual might carry. This suggests that with a complete B-cell repertoire, there might actually be a redundant level of motif matching across all Human Proteome motifs. The bias of $\mathrm{IgV}$ matches toward the more commonly used motifs is likewise seen in the Pathogens (11.5\%) and in the Microbiome (10.7\%). Thus in each proteome, while the IgV match seems to favor the more commonly encountered motifs, which are present more often than 1 in $1024 \mathrm{IgV}$ clonotypes, the somatic hypermutation process is evidently capable of generating a range of motifs beyond what are found in the TCEM of the Human and bacterial proteomes.

We hypothesized that commonly used pentamers might serve some consistent structural or functional role in proteins. If so, then the relative frequency of occurrence of particular pentamer motifs would be the same regardless of the source organism as motifs that serve critical physicochemical roles in protein structure would be expected to occur in either bacteria or man at similar frequencies. To evaluate this we computed the overall rank correlation of usage frequency in the different proteomes (Table 4). The Spearman rank correlation in motif usage between Microbiome and Pathogen proteomes is 0.7. Although quite high, it is clearly substantially less than unity. The rank correlation was lower still between either of the bacterial sets and the Human proteome at approximately 0.5 indicating that human and bacteria comprise different patterns of TCEM in building proteins. The rank correlation between IgHV and IgLV motif usages is 0.27 but the correlations of usage between the $\mathrm{IgV}$ and all of the proteomes is very low (0.03-0.06), indicating no structural or functional relationship of $\operatorname{IgV}$ to the proteome sets.

\section{Missing Motifs}

There are 121,683 motifs of the 3.2 million possible that are absent from all three proteome datasets (Table 1; Figure 2). The amino acid composition of this set of "forbidden" motifs comprises high 


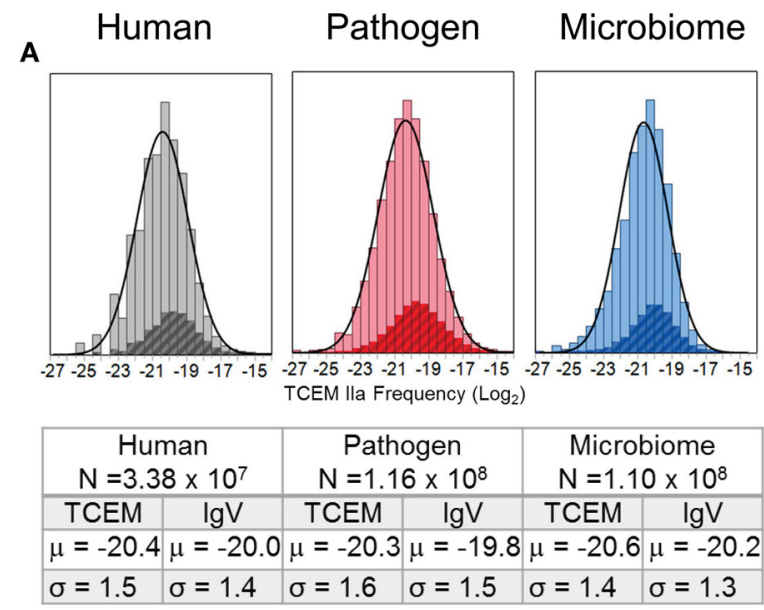

B
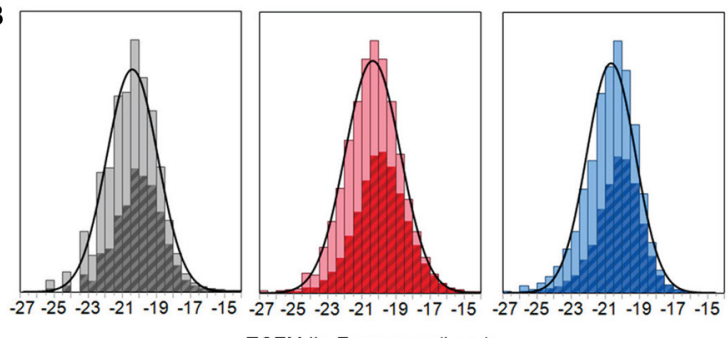

TCEM lla Frequency $\left(\log _{2}\right)$

C

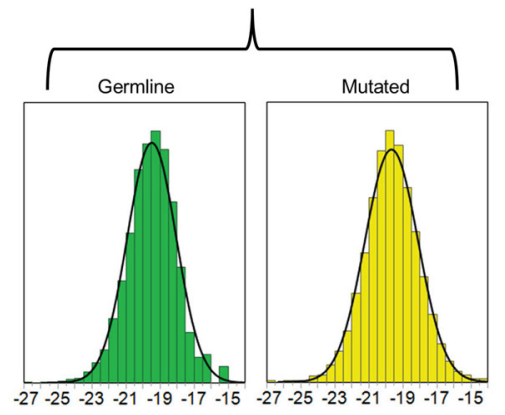

FIGURE 3 | Histograms of TCEM Ila in each proteome set analyzed. (A) The frequency of occurrence of each of the possible $20^{5}$ TCEM Ila pentamers was computed for all of the proteins in each of the proteomes. The fit of a frequency-weighted normal distribution to each of the datasets is shown by the solid curve. The number of motifs and the parameters of the normal distribution curve are given in the inset. The cross hatched region within the histogram corresponds to the frequency of IgV-origin TCEM Ila. $X$ axis shows the $\log 2$ of frequency of occurrence. (B) The background frequency distribution is as in (A) but showing the overlap of IgV TCEM Ila pentamers when a single conservative amino acid replacement is permitted. (C) Separation of the IgV origin pentamers for the Pathogen proteomes to show germline-origin and somatic hypermutated-origin subset distributions of the IgV TCEM Ila.

percentages of tryptophan, methionine, cysteine, and histidine (Figure 5A). The overall coverage of these missing motifs by motifs found in the $\operatorname{IgV}$ dataset is $2.4 \%$ or $2900 / 121,683$ and TCEM of all IgV FC are represented. Again, a complete B-cell repertoire would likely provide more complete coverage of even these rare motifs. Hence, the mutational process generating the $\mathrm{IgV}$ can create even this "forbidden" subset of TCEM that are not

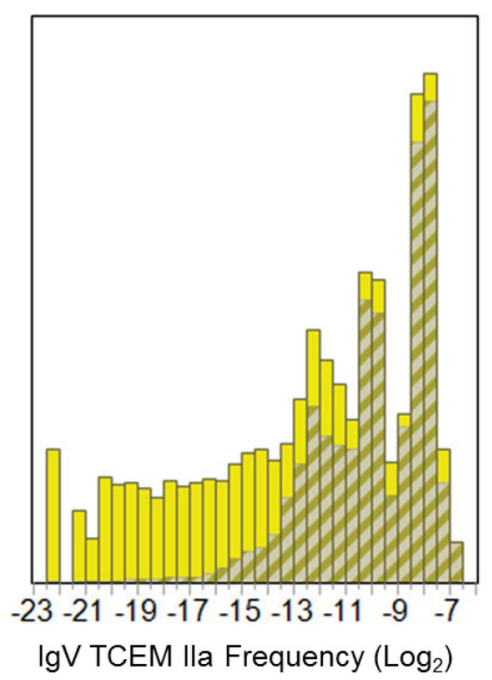

FIGURE 4 | Frequency distributions of IgV-origin TCEM Ila motifs for the Pathogen proteome. Shows the frequency distribution of the IgV TCEM Ila motifs in the Pathogen proteome set as shown by the cross hatched insets in Figure 3A. The bars in the histogram represent the fraction of the total motifs at the particular frequency. The crosshatched region depicts the fraction of the total at the particular frequency that is of germline-origin and the solid portion of the bar is attributable to somatic hypermutation origin motifs. $X$ axis shows the $\log 2$ of frequency of occurrence.

TABLE 3 | Shannon entropy of TCEM Ila motifs in different proteome subsets.

\begin{tabular}{lc}
\hline Source & Shannon entropy $(\boldsymbol{H})$ \\
\hline Human and pathogen & 20.3 \\
Human and microbiome & 20.6 \\
Human & 20.4 \\
IgVL & 12.3 \\
IgVH & 12.5 \\
\hline
\end{tabular}

found in the Human proteome or in the very diverse bacterial proteome datasets.

\section{TCEM Frequency Patterns within the Bacterial Datasets}

Although at a global level the individual bacterial proteomes making up the bacterial datasets are quite similar in TCEM occurrence frequency, there are clear differences in detail of the constituent bacterial proteomes that may be of immunological significance. We observed two independent types of patterns of TCEM occurrence in individual proteins in the bacterial sets:

(a) some genera comprise many proteins with a high percentage of IgV motif overlap, and

(b) each bacterial genus and some species exhibit a characteristic signature of motif usage and of motif overlap with the Human proteome. 
TABLE 4 | Rank correlation of TCEM Ila occurrence between different proteomic subsets.

\begin{tabular}{|c|c|c|c|}
\hline Variable & By variable & Spearman $\rho$ & Prob $>|\rho|$ \\
\hline log2 Human freq & log2 Microbiome freq & 0.5249 & $<0.0001$ \\
\hline log2 Pathogen freq & log2 Microbiome freq & 0.7404 & $<0.0001$ \\
\hline log2 Pathogen freq & $\log 2$ Human freq & 0.5060 & $<0.0001$ \\
\hline $\log 2$ lgVL freq & log2 Microbiome freq & 0.0621 & $<0.0001$ \\
\hline $\log 2 \lg V L$ freq & $\log 2$ Human freq & 0.0652 & $<0.0001$ \\
\hline $\log 2$ lgVL freq & $\log 2$ Pathogen freq & 0.0512 & $<0.0001$ \\
\hline $\log 2 \lg V H$ freq & log2 Microbiome freq & 0.0496 & $<0.0001$ \\
\hline $\log 2 \lg V H$ freq & $\log 2$ Human freq & 0.0382 & $<0.0001$ \\
\hline $\log 2 \lg V H$ freq & $\log 2$ Pathogen freq & 0.0372 & $<0.0001$ \\
\hline $\log 2$ lgVH freq & log2 IGVL freq & 0.2699 & $<0.0001$ \\
\hline
\end{tabular}
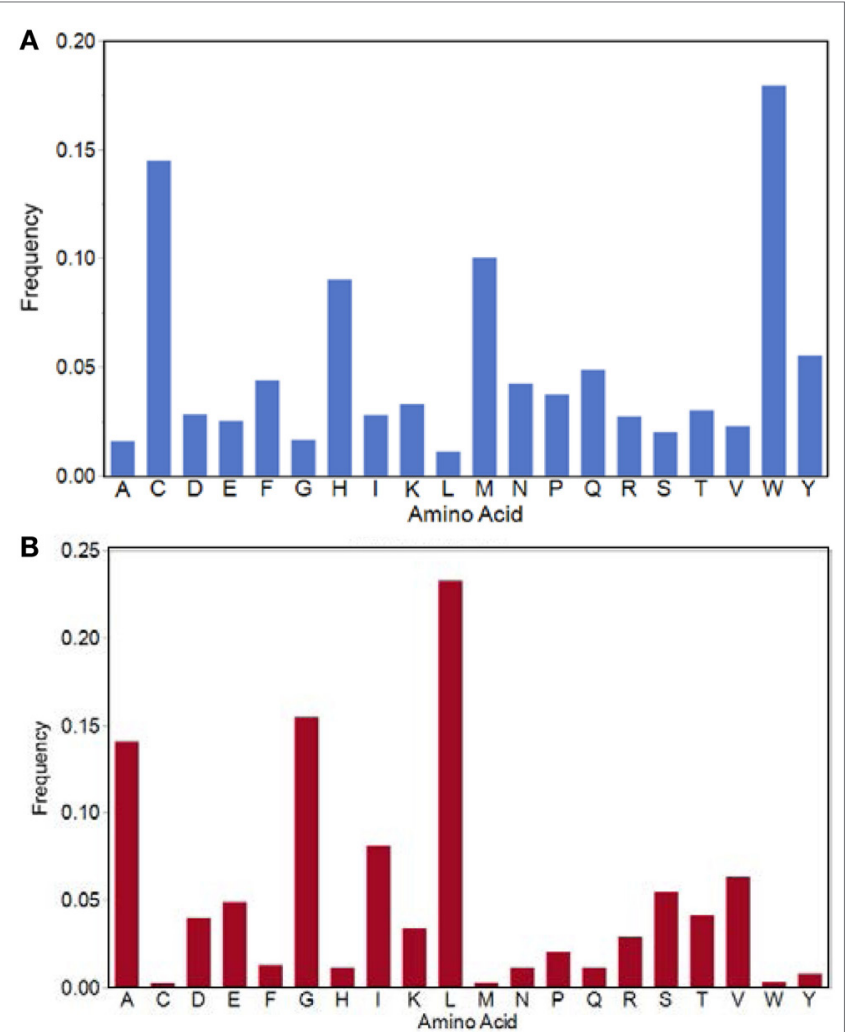

FIGURE 5 | Amino acid frequencies in the TCEM Ila pentamers. (A) Amino acid frequency in the potential motifs which are not found in any of the proteomes evaluated. (B) Amino acid frequencies in the motifs used by all bacteria in both Pathogen and Microbiome sets and the Human proteome.

\section{Comparison of IgV TCEM Content in Pathogen and Microbiome Proteomes}

As shown in Figure 6, when the proteomes of each bacterial Pathogen genus analyzed are arrayed by their IgV motif content, there are clear differences between genera and between species and the IgV motif found in the Human proteome (see also Table S6 in Supplementary Material for analysis of variance). In particular, Burkholderia spp and Mycobacterium spp exhibit a large number of proteins with up to $50 \% \mathrm{IgV}$ motif content. As a group, the majority of the selected Pathogens have a higher content of TCEM shared with IgV than does the Human proteome. In Burkholderia spp and Mycobacterium spp in particular there are entire families of proteins that have high IgV similarity. The high IgV TCEM content is found in individual proteins and in classes of proteins sharing functional similarities. Proteins of some Pathogen genera have a lower overall IgV motif content, but each has a small number of proteins that are outliers with respect to their $\mathrm{IgV}$ motif content. As Figure 6 also indicates (see coloration) these outliers with higher IgV match include quite large proteins (>2000 amino acids) and $\operatorname{IgV}$ content is expressed as a percentage of TCEM, so their size might be expected to significantly contribute to their overall immunological effect.

In contrast, applying a similar analysis to the Microbiome set (Figure 7; Table S7 in Supplementary Material) does not reveal differential distribution with respect to $\mathrm{IgV}$ motif content. All of the genera have similar patterns, although notably Escherichia, included in the Microbiome set, does have a slightly higher content of IgV motifs. In part the lack of distinct patterns among the Microbiome maybe attributable to the taxonomic diversity, however the $\operatorname{IgV}$ motif content is overall similar to the lower range seen in the Pathogen set.

To relate the $\mathrm{IgV}$ content to Human proteome content we applied the frequency-weighted distributions for TCEM as shown in Figure 2A. Each TCEM in the proteins of the proteomes of Figure 6 was assigned its standardized frequency ( $\sigma$ position on the normal distribution curve in the Human proteome shown in Figure 3A). Then, this TCEM metric was averaged across each protein to give each protein an overall score, essentially a "human-like" score for an entire protein. As an example Figure S8 in Supplemental Material shows how the IgV and human score compare for B. pseudomallei. The patterns demonstrate that the high frequency IgV-origin TCEM also indicates an unusually high content of Human proteome motifs.

\section{Organism-Specific Motif Signatures}

We next addressed the genus-specific occurrence of TCEM within the proteome datasets by using treemapping to examine the intraset differences in presence and absence of motifs. The treemaps each depict the underlying patterns of occurrence over $10^{8}$ TCEM. The graphics each contains between 1.5 and 2.5 million rectangles. Each rectangle represents a unique TCEM sharing relationship of motifs among bacterial species and is sized to represent the number of motifs within that combination. The treemap for the Pathogen data set is shown in Figure 8. Figure S12 in Supplementary Material shows magnification of a small area of the upper left corner of Figure $\mathbf{8}$ to show the underlying detail of motif sharing relationships. Strikingly, within the very large Pathogen dataset there are a number of relatively large rectangles visible in Figure 8. Each of the large rectangles contains a motif combination, or signature, that is unique to one or more species of a single genus. For instance, Streptococcus pneumoniae (Box 19) has 6,568 TCEM IIa pentamers not found in the other 131 species analyzed. The top 20 in each of the Pathogen proteomes is indicated in the table accompanying Figure 8. Each of these 20 rectangles each represents $0.84-4.05 \%$ of the total proteome of an organism. Thus, each organism contains a subset of TCEM that 


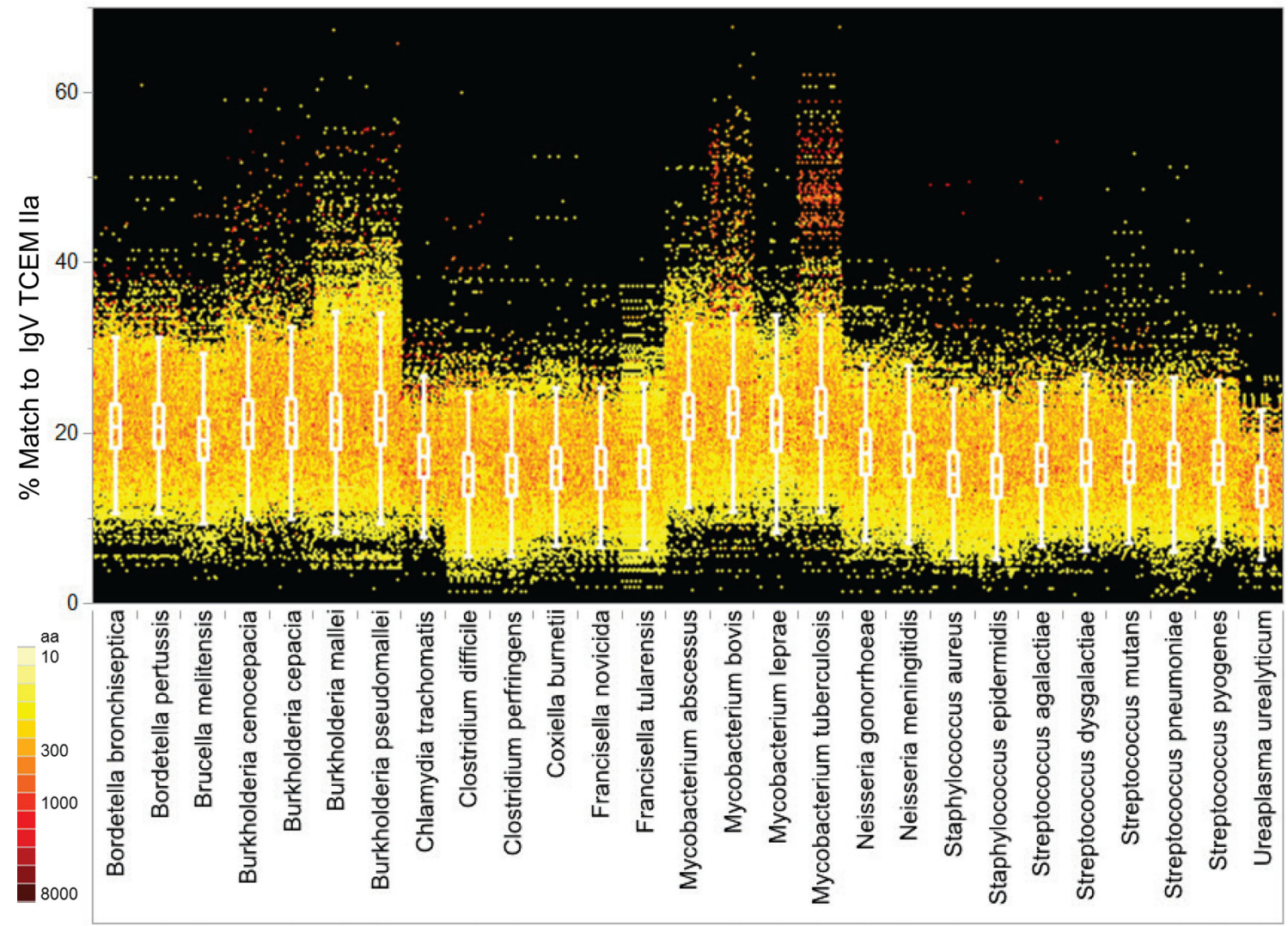

FIGURE 6 | IgV TCEM Ila frequency patterns within the Pathogen proteomes. $X$ axis: groups of five Pathogen total proteomes are clustered by genus with each protein represented by a colored dot. $Y$ axis shows the percentage match of IgV TCEM lla in each protein. Each dot represents the metric computed for a single protein in the proteome of the different organisms. The color of the dot is proportional to the molecular weight of the protein. For example, Mycobacterium tuberculosis has a number of relatively large proteins which have nearly a $50 \%$ match to the lgV motifs. The inset box shows the interquartile range of the metric in each of the proteomes and the whiskers indicate the 10 and $90 \%$ of the metric. For the human proteome the average content of IgV TCEM Ila is $17.3 \%$.

are unique to itself and that in some cases comprises a significant fraction of its entire proteome. There are also sets of motifs that are universally shared or nearly so, shown in dark red boxes in the upper left corner of the treemap. These are the motifs that are found in every bacterial species in the set.

When the Pathogen set was then combined with the Human proteome it emerges that that nearly every bacterial species has a subset of its genus-specific TCEM that it shares with the Human proteome (Figure 9). This overlap is to be expected based on the general overlaps shown in Table 1. In Figure 9, the TCEMs unique to the Human proteome and not found in any of the 132 proteomes of the Pathogen set are represented in the largest rectangle. The other large rectangles identify motifs shared by Human proteome and one or more Pathogens as indicated in the Table in Figure 9. For example, Coxiella burnetti (Box 12) has 7,792 motifs uniquely shared with the Human. A human would obviously not encounter all of the Pathogens simultaneously and the patterns seen when the Human proteome is compared to any one of the Pathogens is itself quite striking. Figure 5B shows the amino acid composition of the 615 motifs shared among Human and both bacterial proteome sets. These motifs favor leucine, glycine, alanine, and isoleucine.
Figure 10 shows the corresponding patterns of unique bacterial signatures for the Microbiome bacteria analyzed. Here the unique signatures tend to constitute a smaller fraction of the individual proteomes, from 0.66 to $1.7 \%$, however this may be a reflection of the greater diversity of genera in this dataset. Figure $\mathbf{1 1}$ shows the unique patterns of sharing with the Human proteome of TCEM in the Microbiome.

To illustrate how identical TCEM may be associated with multiple different GEMs and thus have different MHC binding affinities in their source protein, a small subset of TCEM shared between Human proteome and Burkholderia pseudomallei (Box 8 of Figure 9) were examined. An example of this comparison is provided in Figure 12. Here it is seen that a single TCEM IIa $\sim \sim \sim \mathrm{HV} \sim \mathrm{R} \sim \mathrm{SR} \sim \sim \sim \sim$ was found in 10 different human proteins and 3 different $B$. pseudomallei proteins. The 15 -mer context is different in each case and this means the predicted MHC binding for each is different. While $\mathrm{DRB} 1^{\star} 01: 01$ binds relatively poorly to all the 15 -mers, $\mathrm{DRB} 1^{\star} 03: 01$ binds more strongly and in particular when the TCEM is in the context of one human protein 15-mer (YWEVHVGRRSRWFLG) and the three B. pseudomallei proteins. While for each allele, the same T-cell would recognize the same TCEM, the cumulative duration and repetition of 


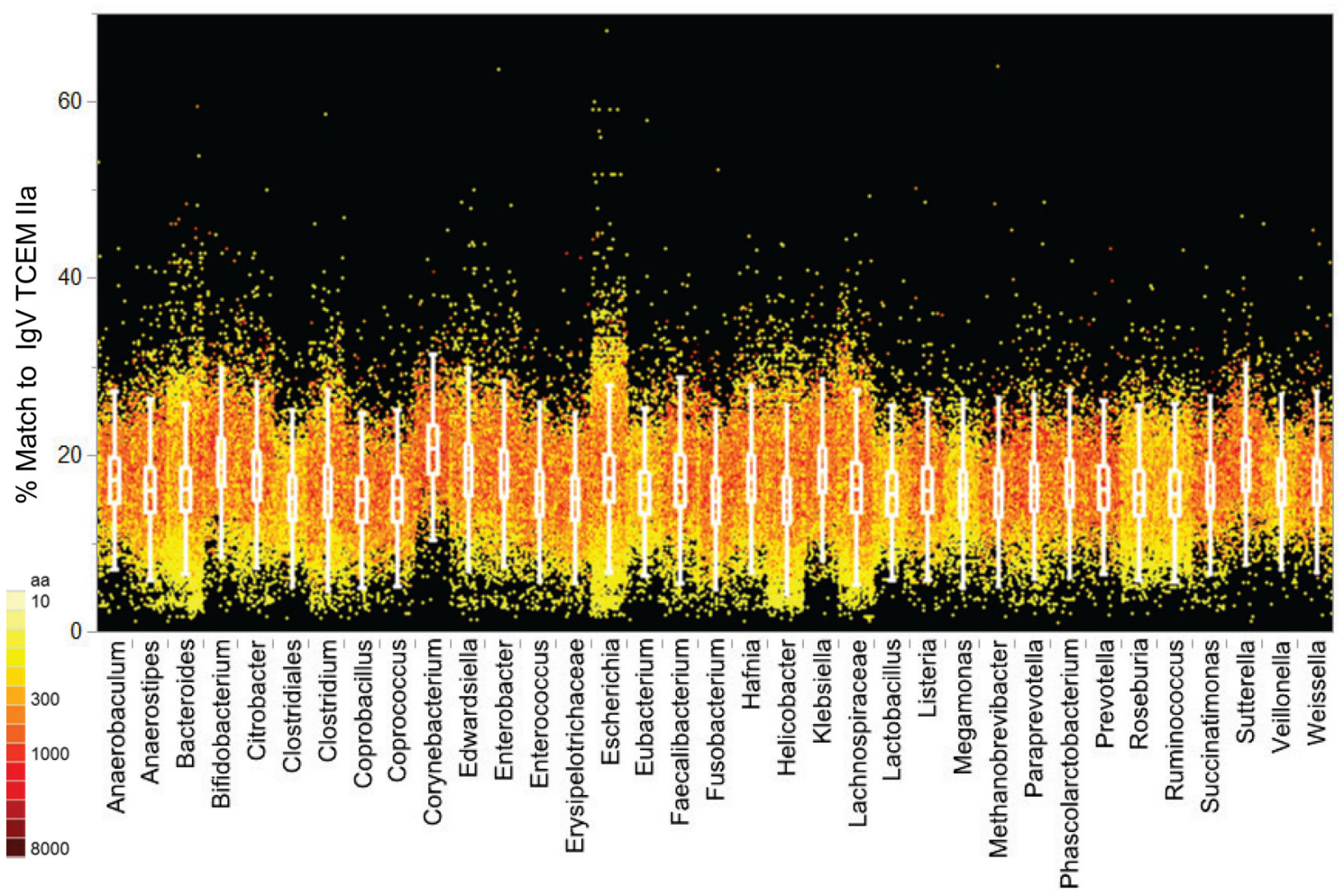

FIGURE 7 | IgV TCEM Ila frequency patterns in the Microbiome proteomes. $X$ axis: groups of Microbiome proteomes are clustered by genus with each protein represented by a colored dot. $Y$ axis shows the percentage match of IgV TCEM Ila in each protein. Each dot represents the metric computed for a single protein in the proteome of the different organisms. The color of the dot is proportional to the molecular weight of the protein. The inset box shows the interquartile range of the metric in each of the proteomes and the whiskers indicate the 10 and $90 \%$ of the metric. For the human proteome the average content of IgV TCEM is $17.3 \%$.

interaction would depend on the effect of the binding affinity on the peptide dwell time in the pMHC complex, and thus the probability of a productive T-cell encounter occurring.

As shown in Figure 3, TCEM frequencies are log-normally distributed and appear to be the result of random processes. Thus, unique motifs might occur at random anywhere in a proteome. However, on closer inspection a substantial number Pathogenunique "signature motifs" appear to cluster in individual proteins, and even in relatively contiguous segments of proteins. A majority of the unique motifs represented in the larger boxes in the treemaps (Figure 8) are found in segments of only 50-100 different proteins in each proteome.

\section{DISCUSSION}

In the present study, we compare two diverse sets of composite bacterial proteomes, which coexist with the human proteome, by using the recognition patterns of the cellular immune system. We also compare the occurrence of the same recognition patterns in $\mathrm{IgV}$ and the human proteome. Genomic comparisons generally focus on analysis of single nucleotide or amino acid changes. By opting to use the TCEMs as the comparator, we are in effect looking how the proteins in the organisms are recognized by the
T-cells as the initiator of any selective pressure by the cellular immune system.

A central challenge in immunology is to understand how self is distinguished from non-self. What emerges from our analysis is that, at least for the T-cell response, this concept is a considerable oversimplification of a complex signaling system. Indeed, what we observe in the present analysis is that what may be uniquely human is, at best, only a very small subset of the possible T-cell recognition signals. When viewed as TCEM pentamers recognized by T-cells in contact pattern IIa and comparing the human proteome to a range of pathogenic and commensal bacteria, only $1.9 \%$ of the possible pentamers are unique to humans. If we consider all three contact pattern of T-cell recognition (TCEM I, IIa and IIb) then the truly unique peptides may be the multiple of these metrics and much smaller. The Microbiome dataset analyzed here is only a small subset of the total gastrointestinal microbiome. An individual human would not be exposed to all of the Pathogens simultaneously. However, over time, the larger the number of bacteria that are encountered, the more closely the number of TCEM unique to humans approaches zero. The organism-specific patterns would be expected to play a role in the overall recognition. Although no single TCEM that an individual $\mathrm{T}$-cell receptor binds apparently defines self, the T-cell repertoire 


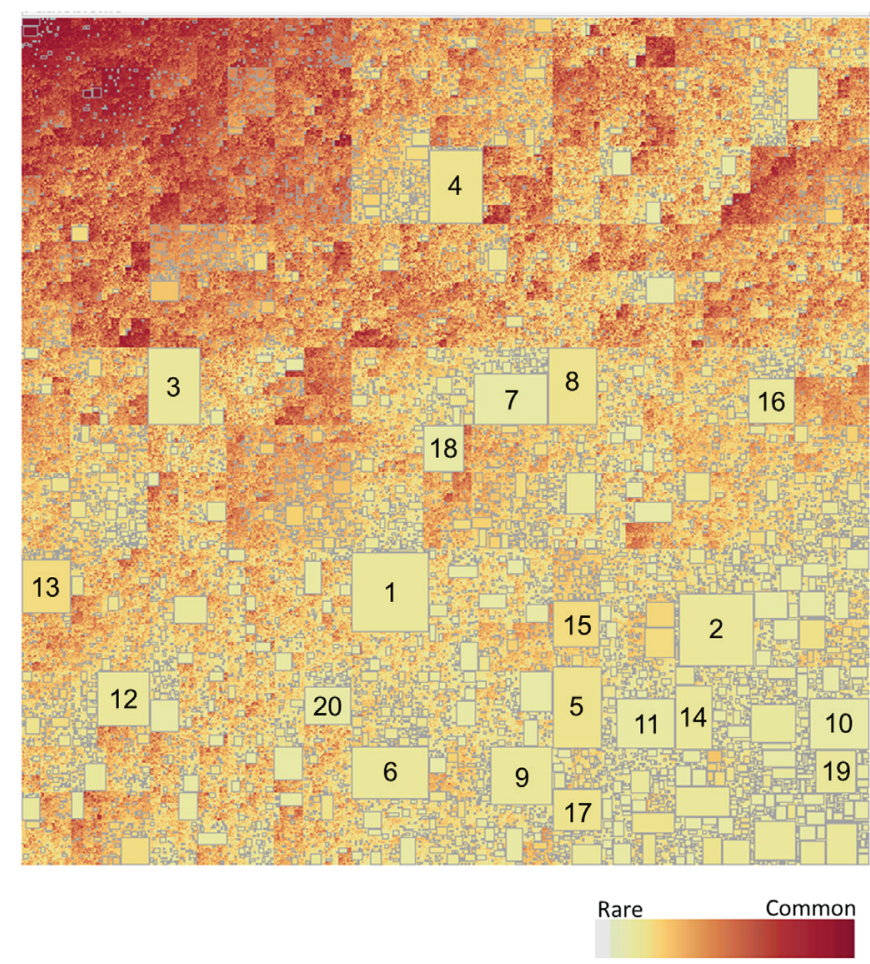

\begin{tabular}{|c|c|c|c|c|}
\hline Box & Genera/Species & $\mathbf{N}$ & $\%$ & $\begin{array}{l}\text { Unique } \\
\text { motifs }\end{array}$ \\
\hline 1 & Clostridium difficile & 5 & $4.05 \%$ & 22,843 \\
\hline 2 & Mycobacterium abscessus & 5 & $3.15 \%$ & 19,567 \\
\hline 3 & Brucella melitensis & 5 & $3.11 \%$ & 15,460 \\
\hline \multirow[t]{2}{*}{4} & Bordetella bronchiseptica & 5 & $2.58 \%$ & \multirow[t]{2}{*}{14,882} \\
\hline & Bordetella pertussis & 5 & $2.90 \%$ & \\
\hline \multirow[t]{2}{*}{5} & Mycobacterium bovis & 5 & $2.79 \%$ & \multirow[t]{2}{*}{14,831} \\
\hline & Mycobacterium tuberculosis & 5 & $2.73 \%$ & \\
\hline 6 & Clostridium perfringens & 5 & $2.99 \%$ & 14,764 \\
\hline \multirow[t]{2}{*}{7} & Burkholderia mallei & 5 & $2.31 \%$ & \multirow[t]{2}{*}{14,171} \\
\hline & Burkholderia pseudomallei & 5 & $1.98 \%$ & \\
\hline 8 & Burkholderia cepacia & 1 & $1.75 \%$ & 13,926 \\
\hline 9 & Coxiella burnetii & 5 & $3.50 \%$ & 13,053 \\
\hline 10 & Staphylococcus epidermidis & 1 & $2.47 \%$ & 10,787 \\
\hline 11 & Mycobacterium leprae & 2 & $2.75 \%$ & 10,555 \\
\hline \multirow[t]{2}{*}{12} & Burkholderia cenocepacia & 3 & $1.47 \%$ & \multirow[t]{2}{*}{10,476} \\
\hline & Burkholderia cepacia & 2 & $1.36 \%$ & \\
\hline \multirow[t]{4}{*}{13} & Burkholderia cenocepacia & 3 & $1.41 \%$ & \multirow[t]{4}{*}{9,996} \\
\hline & Burkholderia cepacia & 2 & $1.30 \%$ & \\
\hline & Burkholderia mallei & 5 & $1.63 \%$ & \\
\hline & Burkholderia pseudomallei & 5 & $1.39 \%$ & \\
\hline 14 & Staphylococcus aureus & 5 & $1.87 \%$ & 8,854 \\
\hline \multirow[t]{2}{*}{15} & Francisella novicida & 5 & $2.16 \%$ & \multirow[t]{2}{*}{7,997} \\
\hline & Francisella tularensis & 10 & $2.31 \%$ & \\
\hline 16 & Chlamydia trachomatis & 5 & $3.55 \%$ & 7,949 \\
\hline \multirow[t]{2}{*}{17} & Neisseria gonorrhoeae & 5 & $1.99 \%$ & \multirow[t]{2}{*}{7,489} \\
\hline & Neisseria meningitidis & 5 & $1.97 \%$ & \\
\hline 18 & Burkholderia cepacia & 1 & $0.98 \%$ & 7,312 \\
\hline 19 & Streptococcus pneumoniae & 5 & $1.67 \%$ & 6,568 \\
\hline 20 & Burkholderia cenocepacia & 1 & $0.84 \%$ & 6,369 \\
\hline
\end{tabular}

FIGURE 8 | Treemap of TCEM Ila frequency patterns in the Pathogen proteomes. Each organism shows a different pattern of occurrence of the possible $20^{5}$ total motifs. This is a binary absence/presence depiction analogous to a Venn diagram. It comprises 1,540,176 different patterns of 110 million motifs. To construct this map tallies were made of each of the TCEM lla motifs found in each organism's proteome. A binary coding scheme was used to score the presence or absence of a particular motif (of $20^{5}$ total) in a proteome. The size of the rectangle is proportional to the number of motifs. The lightly colored rectangles indicate relatively rare motifs and the dark rectangles indicate common motifs. The large rectangles indicate motifs unique to one or a few species; the organism corresponding to each large rectangle is shown in the accompanying tabulation along with the number of motifs in each of the $\mathrm{N}$ proteome and the fraction of the total of each proteome of the organism represented by those motifs. Not visible at this resolution in the upper left hand, darkly colored area, there is a rectangle comprising 615 "universal" motifs that are found in every proteome in this set.

as a whole is capable of uniquely recognizing the overall composite patterns.

The TCEM does not function alone in determining the outcome of a T-cell-peptide interaction, but is recognized within the context of the surrounding MHC histotope. An individual host has a limited set of MHC alleles, which can present a peptide and thus form the surrounding histotope surface. Furthermore, the binding affinity of the inner face of the MHC-bound peptide, the GEM, determines the dwell time of the peptide in the MHC groove and hence the probability of productive T-cell:pMHC encounter, the duration of any T-cell interaction, or how many times it is repeated. Here again an individual's HLA alleles are determinants of the T-cell response. Thus, both "faces" of the peptide, T-cell exposed and groove exposed, modulate the overall outcome in the context of the host's immunogenetics $(19,20)$. One example of this is provided in Figure 12.

The diversity in possible GEMs is also vast. In the case of MHC I, the GEMs comprise $20^{4}$ potential combinations (assuming a 9-mer peptide), and for MHC II a further $20^{10}$ (assuming a 15 -mer) combinations exist. These $20^{10}$ variants in GEM are multiplied by the potential 3.2 million different TCEMs to arrive at the maximum number of potential specific interactions of peptides with T-cells in each of the three contact patterns. GEMs determine on-off rate, or duration, of peptide MHC binding. However, it is the TCEM, and the frequency of its occurrence, which determines the size of the cognate T-cell population responding to the peptide and which can hence contribute to 


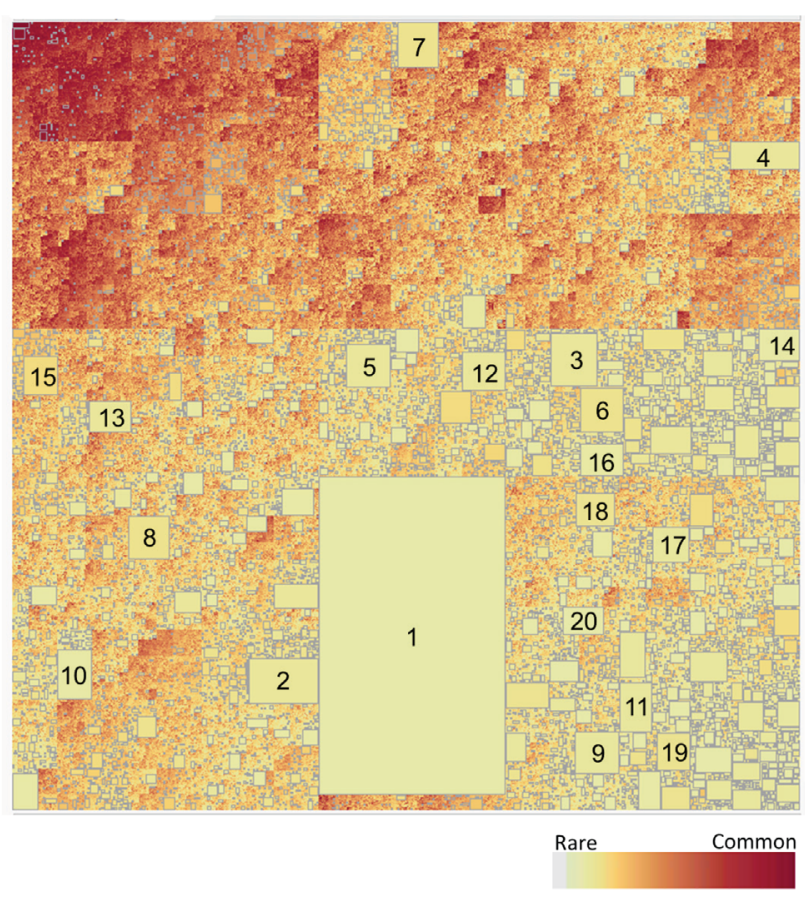

\begin{tabular}{|r|l|r|}
\hline Box & \multicolumn{1}{|c|}{ Genera } & $\begin{array}{c}\text { Unique } \\
\text { motifs }\end{array}$ \\
\hline 1 & Human & 275,518 \\
\hline 2 & Human / Clostridium difficile & 14,189 \\
\hline 3 & Human / Mycobacterium abscessus & 11,714 \\
\hline 4 & Human / Brucella melitensis & 9,244 \\
\hline 5 & Human / Clostridium perfringens & 9,145 \\
\hline 6 & Human / Mycobacterium bovis / Mycobacterium & 8,896 \\
\hline 7 & tuberculosis & \\
\hline 8 & Human / Bordetella bronchiseptica / Bordetella pertussis & 8,814 \\
\hline 9 & Clostridium difficile & 8,726 \\
\hline 10 & Human / Burkholderia cepacia & 8,654 \\
\hline 11 & Mycobacterium abscessus & 8,253 \\
\hline 12 & Human / Coxiella burnetii & 7,853 \\
\hline 13 & Human / Burkholderia cenocepacia / Burkholderia cepacia & 7,792 \\
\hline 14 & Human /Staphylococcus epidermidis & 6,438 \\
\hline 15 & Human / Burkholderia cenocepacia / Burkholderia cepacia & 6,404 \\
\hline & Burkholderia mallei /Burkholderia pseudomallei & 6,397 \\
\hline 16 & Human / Mycobacterium leprae & 6,377 \\
\hline 17 & Brucella melitensis & 6,216 \\
\hline 18 & Bordetella bronchiseptica / Bordetella pertussis & 6,068 \\
\hline 19 & Mycobacterium bovis / Mycobacterium tuberculosis & 5,935 \\
\hline 20 & Burkholderia cepacia & 5,673 \\
\hline &
\end{tabular}

FIGURE 9 | Treemap of combined TCEM Ila frequency patterns in the Pathogen proteomes and the Human proteome. The algorithm is the same as used in Figure $\mathbf{7}$ but in this case the human proteome was also included in the analysis. This shows the inter-relationship between the motifs of the Pathogen and the Human. It comprises 1,585,188 different motif sharing patterns. The accompanying tabulation of the 20 largest boxes indicates the composition depicted in these rectangles. For example, Box 1 shows that there are 275,510 motifs are unique to humans and not found in any of the pathogens whereas Box 2 shows the number of motifs that are found in both the $C$ difficile and Human proteomes and indicates that 14,189 of the 22,843 unique Pathogen motifs found in the organism (Figure 7) are also found in the Human proteome. Overall, this shows a remarkable overlap between the T-cell motifs found in the proteins of pathogens and those found in humans.

T-cell repertoire phenotypes and the balance of immune stimulation or suppression $(21,22)$.

Common TCEM, like those found repeatedly in IgV, provide for a higher frequency of interaction with cognate T-cells. Higher signal strength generated through a combination high frequency pMHC:T-cell contact and longer interaction dwell time favors emergence of a Treg phenotype, through a combination of Foxp3 induction (23) and epigenetic changes in the T-cells (24-26). Higher affinity MHC binding of the peptide GEM ensures a longer dwell time and thus more opportunity for interactions at the T-cell exposed face. Implicitly, high frequency TCEMs may build up a larger cognate T-cell population. So provided they also occur in peptides which bind well to the MHC, high frequency TCEMs will therefore be expected to generate more Tregs. The balance of Treg and T-effectors is modulated by IL2 secretion by T-effectors and binding and removal of IL2 by Treg (27-29). Local binding of IL2 will accelerate Treg proliferation, suppressing effector responses in the vicinity and potentially leading to greater numbers of residual cells of the same specificity available for future interaction (30). Such a feedback loop driven by the frequency of TCEM appearance is consistent with a form of quorum sensing among T-cells (31). It also indicates that down-regulation and homeostasis is likely the norm driven by common TCEM. Conversely, the lower frequency motifs are likely to be more important in discriminating between self and non-self and in generating an upregulated immune response.

A further consideration is the abundance of the protein in which a TCEM is located, how frequently it is transcribed and catabolized, and its accessibility to antigen presenting cells. In the case of bacterial proteomes, these factors will change either as different proteins are expressed with the stage of infection or with changes in the microbial ecology of the microbiome. Interestingly, this may make the modulating role of immunoglobulin-derived peptides relatively more important as immunoglobulins are constantly accessible, readily processed, and presented by B cells.

While we have interpreted the exposure of amino acids in TCEMs in three simple contact patterns based on the structural analysis of Rudolph et al. (2), flexibility of peptides may accommodate some bulging and twisting of MHC-bound peptides to create some variability in the TCEM exposure (32) and so we expect that the interaction will be much more nuanced. Additionally other studies have examined the relative importance of each amino acid in the binding groove (33). A further compounding factor is that there may be multiple clonal 


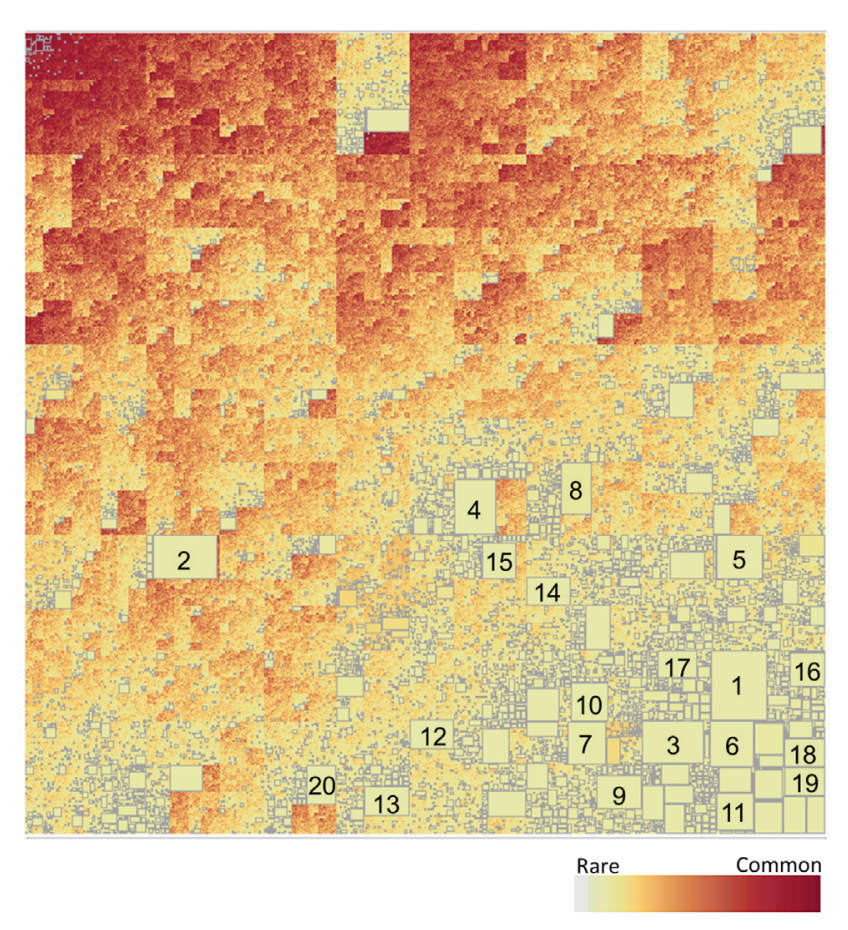

\begin{tabular}{|r|l|r|r|}
\hline Box & \multicolumn{1}{|c|}{ Organism } & $\%$ & $\begin{array}{c}\text { Unique } \\
\text { motifs }\end{array}$ \\
\hline 1 & Lachnospiraceae bacterium 3_1_57FAA_CT1 & $1.69 \%$ & 17,918 \\
\hline 2 & Bacteroides cellulosilyticus DSM 14838 & $1.29 \%$ & 13,379 \\
\hline 3 & Lachnospiraceae bacterium 7_1_58FAA & $1.48 \%$ & 12,264 \\
\hline 4 & Bacteroides sp. 3_1_19 & $1.20 \%$ & 10,474 \\
\hline 5 & Erysipelotrichaceae bacterium 21_3 & $1.29 \%$ & 9,519 \\
\hline 6 & Paraprevotella xylaniphila YIT 11841 & $1.29 \%$ & 9,398 \\
\hline 7 & Hafnia alvei ATCC 51873 & $0.99 \%$ & 7,901 \\
\hline 8 & Bacteroides sp. D22 & $0.78 \%$ & 7,528 \\
\hline 8 & Bacteroides xylanisolvens SD CC 1b & $0.78 \%$ & \\
\hline 8 & Bacteroides xylanisolvens SD CC 2a & $0.79 \%$ & \\
\hline 9 & Klebsiella pneumoniae subsp.pneumoniae WGLW3 & $0.84 \%$ & 6,976 \\
\hline 10 & Faecalibacterium prausnitzii M21/2 & $1.14 \%$ & 6,547 \\
\hline 11 & Prevotella oralis HGA0225 & $1.11 \%$ & 6,365 \\
\hline 12 & Coprobacillus sp. 8_2_54BFAA & $0.94 \%$ & 6,270 \\
\hline 13 & Clostridium sp. M62/1 & $0.89 \%$ & 6,123 \\
\hline 14 & Edwardsiella tarda ATCC 23685 & $0.98 \%$ & 6,021 \\
\hline 15 & Citrobacter youngae ATCC 29220 & $0.70 \%$ & 5,789 \\
\hline 16 & Lachnospiraceae bacterium 5_1_57FAA & $0.85 \%$ & 5,325 \\
\hline 17 & Lachnospiraceae bacterium 2_1_58FAA & $0.82 \%$ & 5,272 \\
\hline 18 & Ruminococcus obeum A2-162 & $0.84 \%$ & 5,190 \\
\hline 19 & Sutterella wadsworthensis 2_1_59BFAA & $1.11 \%$ & 5,152 \\
\hline 20 & Bacteroides eggerthii DSM 20697 & $0.66 \%$ & 5,139 \\
\hline & & \\
\cline { 2 - 3 } & & & \\
\hline
\end{tabular}

FIGURE 10 | Treemap of TCEM Ila frequency patterns in the Microbiome proteomes. See Figure 7 for more complete description. This treemap comprises 2,092,911 different motif sharing patterns.

lines of cognate T-cells for any unique TCEM; there is not a one-to-one correspondence of TCEM and T-cell receptor and a T-cell receptor may bind with more than one TCEM-MHC combination, through recognition of physicochemical near neighbors $(16,17)$.

\section{Comparative Overlaps in Motifs Among Proteomes Analyzed}

The bacteria selected for inclusion in the Pathogen set comprised human pathogens, which are generally directly transmitted, not vector borne. With exception of the Clostridium spp, we excluded genera that are typically fecal-oral transmission. This was intended to maintain a clear distinction from the array of bacteria included in the gastrointestinal Microbiome group. Similarly, we opted to include Escherichia spp in the Microbiome set. Such decisions about opportunist pathogens certainly affected the degree of overlap we observed, but do not detract from the overall conclusions. It is noteworthy that E. coli is an outlier among the Microbiome set in Figure 7 with respect to its IgV TCEM content. This is consistent with the prior observations that $E$. coli contains a number of immunoglobulin domains (34).

As bacteria are constantly evolving at different rates, and microbiome composition is affected by external factors, the isolates from which we analyzed sequences simply represent a cross sectional sample or "snapshot" of a dynamic process. Had different bacterial isolates been incorporated into the analysis, results would have differed in detail, but likely not the overall conclusions. Similarly, by selecting the gastrointestinal microbiome, we do not underestimate that the microbiota of other mucosal surfaces exercise a similar relationship with the human proteome and $\mathrm{IgV}$ and undoubtedly also influence immune modulation (35).

In the analysis conducted, we demonstrate that there are different and distinctive patterns of TCEM occurrence in the three proteome groups. Interestingly, the Human proteome utilizes only about three quarters of the possible pentamers and has a relatively small set of 59,256 unique motifs not found in the Microbiome and Pathogen proteomes analyzed. Both bacterial proteome sets we examine overlap extensively with the Human proteome. Slightly more overlap occurs with the resident gastrointestinal Microbiome perhaps indicative of a longer homeostatic coexistence.

This high degree of TCEM commonality underscores the impossibility of central tolerance to all self-antigens $(36,37)$. Rather, it argues for functional modulation of peripheral T-cell responses to specific TCEM along a spectrum of Treg to T-effector, enabled by T-cell plasticity of phenotype (38, 39 ), and the localization of responses in response to mucosal microbiome or to infection (40-42).

While there appears to be no overriding requirement for any given motif driven by basic protein structure, there is a fairly large set of almost 121,683 motifs, which are absent from all three proteome groups. These "forbidden" motifs have amino acid compositions in common that are high in cysteine, histidine, methionine and tryptophan (Figure 5A). The side chains of these 


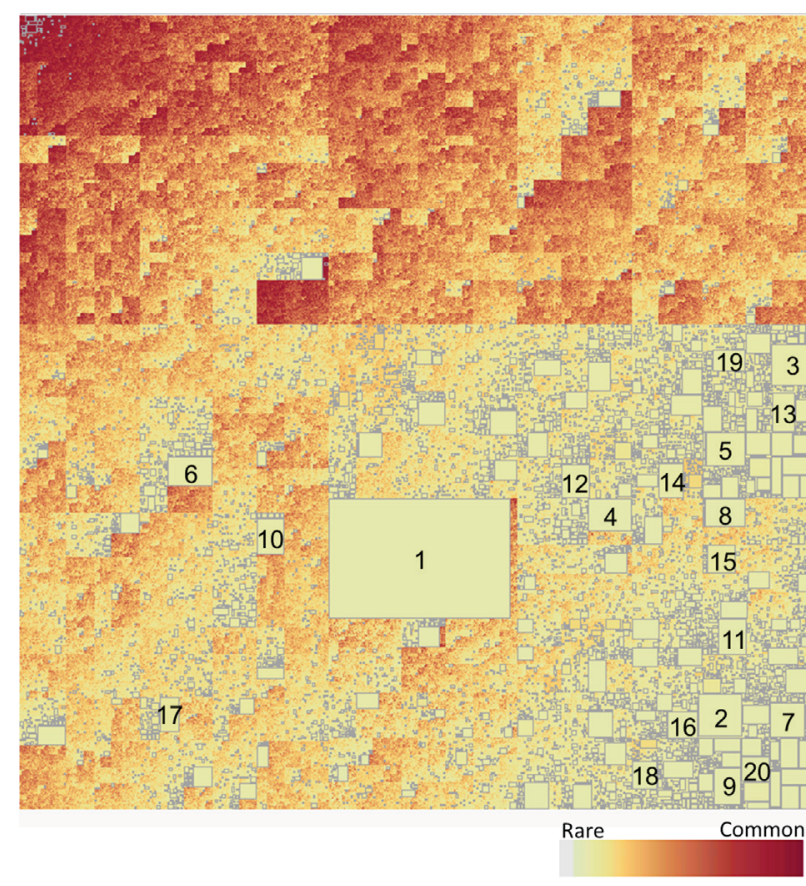

\begin{tabular}{|r|l|r|}
\hline Box & \multicolumn{1}{|c|}{ Organism } & $\begin{array}{c}\text { Unique } \\
\text { motifs }\end{array}$ \\
\hline 1 & Human & 105,831 \\
\hline 2 & Lachnospiraceae bacterium 3_1_57FAA_CT1 & 9,376 \\
\hline 3 & $\begin{array}{l}\text { Human / Lachnospiraceae bacterium } \\
\text { 3_1_57FA_CT1 }\end{array}$ & 8,542 \\
\hline 4 & Bacteroides cellulosilyticus DSM 14838 & 7,062 \\
\hline 5 & Human / Lachnospiraceae bacterium 7_1_58FAA & 6,373 \\
\hline 6 & Human / Bacteroides cellulosilyticus DSM 14838 & 6,317 \\
\hline 7 & Lachnospiraceae bacterium 7_1_58FAA & 5,891 \\
\hline 8 & Bacteroides sp. 3_1_19 & 5,589 \\
\hline 9 & Paraprevotella xylaniphila YIT 11841 & 4,959 \\
\hline 10 & Human / Bacteroides sp. 3_1_19 & 4,885 \\
\hline 11 & Erysipelotrichaceae bacterium 21_3 & 4,851 \\
\hline 12 & Human / Erysipelotrichaceae bacterium 21_3 & 4,668 \\
\hline 13 & Human / Paraprevotella xylaniphila YIT 11841 & 4,439 \\
\hline 14 & Human / Hafnia alvei ATCC 51873 & 3,975 \\
\hline 15 & $\begin{array}{l}\text { Bacteroides sp. D22 / Bacteroides xylanisolvens SD } \\
\text { CC 1b / Bacteroides xylanisolvens SD CC 2a }\end{array}$ & 3,961 \\
\hline 16 & Hafnia alvei ATCC 51873 & 3,926 \\
\hline 17 & $\begin{array}{l}\text { Human / Bacteroides sp. D22 / Bacteroides } \\
\text { xylanisolvens SD CC 1b / Bacteroides xylanisolvens } \\
\text { SD CC 2a }\end{array}$ & 3,567 \\
\hline 18 & Klebsiella pneumoniae subsp. pneumoniae WGLW3 & 3,512 \\
\hline 19 & $\begin{array}{l}\text { Human / Klebsiella pneumoniae subsp. pneumoniae } \\
\text { WGLW3 }\end{array}$ & 3,464 \\
\hline 20 & Prevotella oralis HGA0225 & 3,387 \\
\hline & & \\
\hline
\end{tabular}

FIGURE 11 | Intersection between the Human proteome and the Microbiome. See Figure $\mathbf{9}$ for more complete description. This treemap comprises 2,144,076 different motif sharing patterns.

amino acids each have physicochemical properties that make it unlikely for them to be in close proximity to each other, which may bias against their use. Conversely, there is a group of motifs that are a relatively smaller "universal set" found in all organisms. Amino acid compositions of these TCEM showed a high content of the hydrophobic amino acids known to be commonly found in membrane-spanning helices.

\section{Motif Patterns in Immunoglobulins}

We show that the diversity of peptides generated within immunoglobulin variable regions is broadly representative of the TCEM in all three proteome datasets and our IgV dataset comprises a stratified random sample across the array of all possible TCEM. We see that our database of $56,000 \mathrm{IgV}$ sequences provides a balanced random coverage for both the human proteome and the two bacterial proteome sets we used in our analysis, and even represents the "forbidden" motifs never used in the three proteome groups. Thus, the somatic mutational process that generates immunoglobulin variable regions is evidently capable of generating any possible 9-mer or 15 -mer peptide. As the dataset of immunoglobulins analyzed is only about $1 \%$ of the full complement of $\operatorname{IgV}$ that any individual would carry (12), it appears that the diversity of TCEM within IgV may be able to stimulate and maintain a T-cell repertoire that is capable of responding to all potential T-cell immunogens. This supports the hypothesis that the frequency of motif occurrence in the $\operatorname{IgV}$ may modulate the $\mathrm{T}$-cell repertoire in the face of selective pressure and assist in maintaining homeostasis and memory (1).

Immunoglobulins are unique compared to the rest of the human proteome in their immense diversity and in their turnover rate. A human carries about 3-9 $\times 10^{6}$ unique B-cell clonotypes (12) yielding variable regions which, combining both light and heavy chains, each have approximately 260 unique sequential peptides. In aggregate, therefore, the immunoglobulin of an individual human comprises a billion or more unique peptides yielding TCEMs in each contact pattern. Moreover, the lifespan of a B-cell is estimated to be of 30-60 days (43) and each immunoglobulin about 14 days (44), so there is considerable turnover. At a serum concentration of about $11 \mathrm{~g} / \mathrm{l}$ the diverse mass of $\mathrm{IgV}$ constitutes about $75 \mathrm{~g}$ in an adult human $(45,46)$. In contrast, the remainder of the human proteome comprises approximately 20,000 proteins averaging about 384 amino acids apiece, or about 7 million unique peptides and has a slower rate of turnover. Thus, immunoglobulins constitute a massive, dynamic protein pool with enormous peptide diversity available throughout the body.

The broad representation of motifs within the $\operatorname{IgV}$ with a range of frequency-of-occurrence of about $2^{16}$ and which are being consistently presented to T-cells (6), also implies that at 


\section{A \\ Source

$\begin{array}{ll}\begin{array}{ll}\text { Human } \\ \text { Human } \\ \text { Human }\end{array} & \text { A6NIH7 } \\ \text { Human } & \text { E7EST6 } \\ \text { Human } & \text { H0YFB1 } \\ \text { Human } & \text { O60423 } \\ \text { Human } & \text { Q6UXG8 } \\ \text { Qum414 } \\ \text { Human } & \text { Q8NFY9 } \\ \text { Human } & \text { Q96DT5 } \\ \text { Buman } & \text { Q99569 } \\ \text { B.pseudomallei } & \text { Q9HCM3 } \\ \text { B.pseudomallei } & 19271492 \\ \text { B.pseudomallei } & 19271494 \\ & \end{array}$

Accession Peptide

TCR Ila $(2,3,5,7,8)$
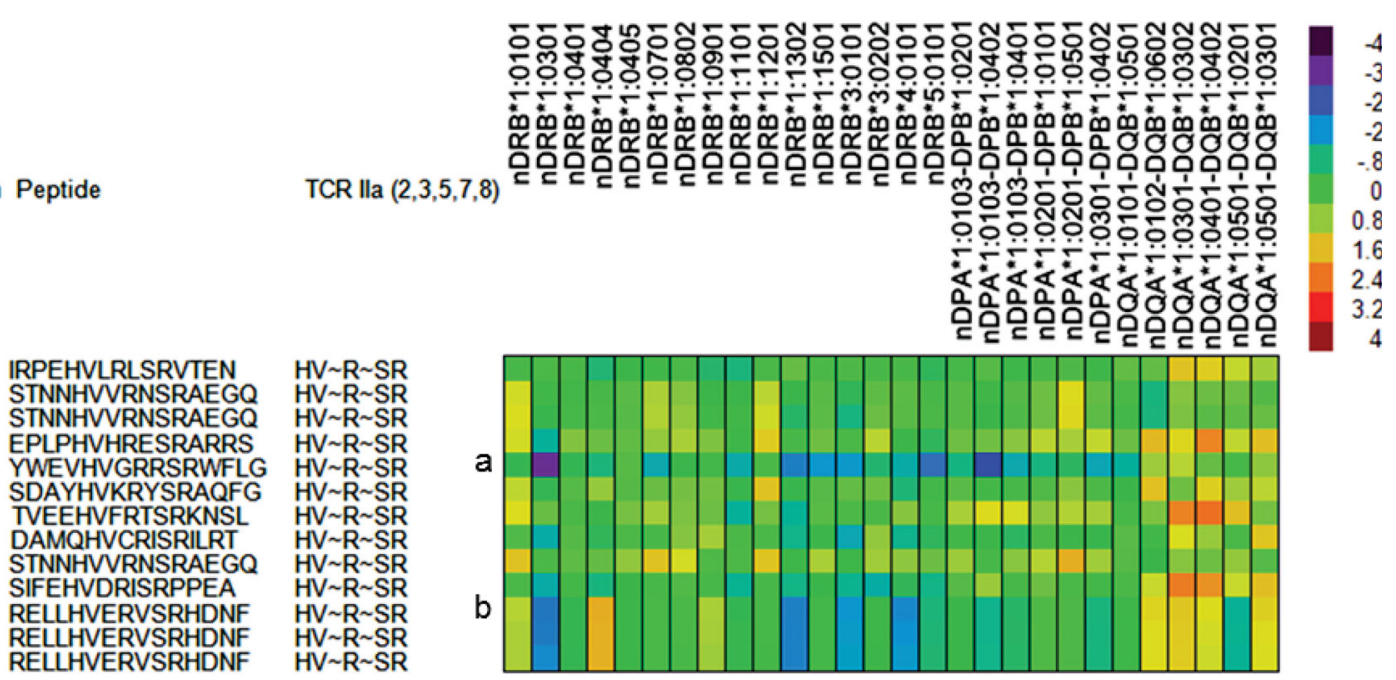

B Source: Q6UXG8 BTNL9_HUMAN Butyrophilin-like protein 9

In source protein predicted binding affinity to DRB1*0301 $=-3.21$

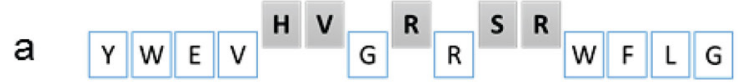

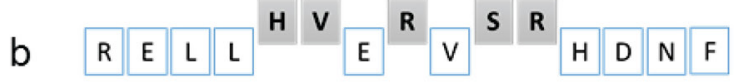

Source: 19271492 : Long-chain-fatty-acid--CoA ligase Burkholderia pseudomallei K96243

In source protein predicted binding affinity to DRB1*0301= -1.81

FIGURE 12 | Differential predicted MHC binding of peptides from human and bacterial proteomes with identical TCEM but different groove exposed motifs. Examples shown are from Box 8 in Figure 9, which comprises 8,726 total motifs. (A) Binding affinities were computed for the MHC II alleles indicated using previously described methods (18) and standardized to zero mean and unit variance within the parent protein. The color scale shows predicted binding in SD units. Source proteins listed by Accession number are listed in full in Table S10 in Supplementary Material. (B) Peptides a and b shown in (A) are shown with the T-cells exposed amino acids and the groove exposed amino acids identified.

any time the T-cell repertoire contains a gradation in frequency of cognate T-cells ready to respond to a fraction of the Pathogen or Microbiome motifs whenever these are first encountered. These may constitute a "starter set" of first responders, which can expand in size and which can be supplemented by respondents to the other motifs. This response would be supplemented by near neighbor matches, in which four of five amino acids generate a near fit $(16,17)$. The sharing of the same T-cell recognition motifs by the human proteome, IgV, and in the Pathogen sets accounts for the observations by others that naïve T-cells sampled from cord blood have the innate ability to recognize pathogens (47, 48). While most observations on cord blood have focused on viral pathogens there is no reason to think they would be more diverse in TCEM usage than bacteria. It is also consistent with the role of the microbiota in maintaining immune homeostasis (35).

The frequency distribution of motif occurrence in each of the three proteome sets is very similar and is log-normal. In contrast, the IgV-mutated set shows a Pareto distribution strongly biased toward the more common motifs (Figure 4). Although any peptide can be generated by the IgV mutational process, within the $\mathrm{IgV}$ data set we examined, half of all the possible TCEM motifs reappear at least once in every $1024 \mathrm{~B}$-cell clonal products (1). This means there is a greater degree of proteome overlap by the high frequency IgV motifs and a lesser overlap by the rare ones. The implication is that more common IgV TCEMs will likely engender larger T-cell populations, more memory, and more Tregs.

Not only is the somatic mutation process capable of generating a highly diverse set of TCEM, the appearance of TCEM in the IgV constitutes a stratified random sample of the TCEM distribution in the Human proteome and the bacterial proteomes. This implies that the TCEM frequency distribution observed in $\operatorname{IgV}$ can likewise be used as a means of characterization of TCEM in proteins from outside of the human proteome.

\section{Patterns Within the Pathogen Set}

Members of the Pathogen set show clear differences in IgV-like motif content in their constituent proteins (Figure 6). Perhaps the most striking feature of this pattern is that two genera, Burkholderia and Mycobacterium, both intracellular organisms that cause chronic diseases, stand out as having many proteins 
that contain motifs used at a high frequency in the IgV. In some proteins the $\mathrm{IgV}$ matched TCEM content is over $50 \%$ of the total TCEM motifs. These two genera also have a high level of uniquely shared motifs with the Human proteome exclusive of $\operatorname{IgV}$ (Figures 8 and 9). Selective advantage may be provided by the camouflage of using very common and likely immunosuppressive motifs. This is consistent with findings reported for Mycobacteria $(49,50)$. Further, in some pathogens, including Mycobacteria, we noted a higher distribution of IgV motifs in proteins that are upregulated early in infection (data not shown), indicating that the spectrum of overlap and immune recognition may change through the infective cycle.

A second striking observation is that, even with the limitation of 3.2 million TCEM motifs, certain subsets of a few thousand motifs were unique to particular bacteria, sometimes down to the species level. This pattern is maintained when the motifs in common with the human proteome are identified. While this pattern will undoubtedly become more diffuse as more organisms are added to the analysis set, it indicates that there are specific signatures that could serve as targets for interventions.

Both these genus- and species-specific patterns point to possible different mechanisms of immune evasion arising from evolutionary pressure. For instance, while Burkholderia and Mycobacterium share many motifs with $\operatorname{IgV}$ and Human proteome and also have fairly large sets of species specific motifs, Francisella is conspicuously absent from the larger clusters of unique signatures seen in Figures 8 and $\mathbf{9}$ and has a lower overall IgV TCEM content (Figure 6). It is not obvious what the evolutionary forces are which give rise to such signature patterns, but their existence suggests an important further line of inquiry toward better understanding of immune evasion and pathogenesis and to provide critical information for vaccine design.

The boxes in Figures 9 and 11, showing the complexity of TCEM overlap between Pathogen or Microbiome organisms with the Human proteome, indicate the extent to which a T-cell responsive to a bacteria may also respond to a human protein. Further examination of this overlap may shed light on how infections or alterations in commensal balance can trigger the onset of autoimmune diseases.

The limitation of 3.2 million TCEMs in each frame accounts for the widely observed T-cell polyspecificity (51-53) and the overall physical limitations on T-cell repertoire size deduced by

\section{REFERENCES}

1. Bremel RD, Homan EJ. Frequency patterns of T-cell exposed amino acid motifs in immunoglobulin heavy chain peptides presented by MHCs. Front Immunol (2014) 5:541. doi:10.3389/fimmu.2014.00541

2. Rudolph MG, Stanfield RL, Wilson IA. How TCRs bind MHCs, peptides, and coreceptors. Annu Rev Immunol (2006) 24:419-66. doi:10.1146/annurev. immunol.23.021704.115658

3. Mason D. A very high level of crossreactivity is an essential feature of the T-cell receptor. Immunol Today (1998) 19(9):395-404.

4. Troy AE, Shen H. Cutting edge: homeostatic proliferation of peripheral Tlymphocytes is regulated by clonal competition. J Immunol (2003) 170(2):672-6. doi:10.4049/jimmunol.170.2.672

5. Sy MS, Benacerraf B. Suppressor T cells, immunoglobulin and Igh restriction. Immunol Rev (1988) 101:133-48. doi:10.1111/j.1600-065X.1988. tb00735.x
Mason (3). The motifs within the total possible array of 3.2 million and, as we indicate here, a somewhat smaller actual utilized repertoire, have to enable recognition of all peptides of self and non-self origin. Unique recognition, and thus initial response as well as recall, depends on the combination of signals, not on a single unique motif, and thus on the aggregate response of multiple T-cell clonal populations.

\section{CONCLUSION}

The analysis of proteomes based on the TCEM contact patterns provides insight into the immune recognition of different classes of organism. Overall, the approach we have used to analyze commonalities and differences provides a new perspective on the discrimination between self and non-self, underscoring that there is very little uniqueness to the individual TCEMs present in the human proteome. Thus, distinguishing self and not self is dependent on a pattern comprised of many TCEM and which includes the relative frequency of each. This analytical approach can be applied more broadly. Any protein, proteome, or collection of proteomes, or mutated neoantigens, can be characterized by their TCEM pentamer content and frequency. This can provide insights and new experimental approaches to autoimmunity, allergies, and other immunopathologies. In the case of the Pathogen set, the TCEM analysis indicates clear differences in motif content between proteins of different genera and species and provides clues to both immune evasion and strategies for intervention, including vaccine design. The breadth and distinct frequency patterns of the immunoglobulin-derived peptides again suggests a role of immunoglobulins in maintaining a broadly responsive T-cell repertoire and memory.

\section{ACKNOWLEDGMENTS}

The authors acknowledge the assistance of Alleen Hager with proteome compilation and proofreading, and the thoughtful comments of Andrea Ferrante.

\section{SUPPLEMENTARY MATERIAL}

The Supplementary Material for this article can be found online at http://journal.frontiersin.org/article/10.3389/fimmu.2015.00538

6. Weiss S, Bogen B. B-lymphoma cells process and present their endogenous immunoglobulin to major histocompatibility complex-restricted T cells. Proc Natl Acad Sci U S A (1989) 86(1):282-6. doi:10.1073/pnas.86.1.282

7. Chakrabarti D, Ghosh SK. Induction of syngeneic cytotoxic T lymphocytes against a B cell tumor. III. MHC class I-restricted CTL recognizes the processed form(s) of idiotype. Cell Immunol (1992) 144(2):455-64. doi:10.1016/0008-8749(92)90259-R

8. Rudensky A, Preston-Hurlburt P, Al-Ramadi BK, Rothbard J, Janeway CA Jr. Truncation variants of peptides isolated from MHC class II molecules suggest sequence motifs. Nature (1992) 359(6394):429-31. doi:10.1038/359429a0

9. Bogen B, Ruffini P. Review: to what extent are T cells tolerant to immunoglobulin variable regions? Scand J Immunol (2009) 70(6):526-30. doi:10.1111/j.1365-3083.2009.02340.x

10. Joao C, Ogle BM, Gay-Rabinstein C, Platt JL, Cascalho M. B cell-dependent TCR diversification. J Immunol (2004) 172(8):4709-16. doi:10.4049/ jimmunol.172.8.4709 
11. AbuAttieh M, Bender D, Liu E, Wettstein P, Platt JL, Cascalho M. Affinity maturation of antibodies requires integrity of the adult thymus. Eur J Immunol (2012) 42(2):500-10. doi:10.1002/eji.201141889

12. Arnaout R, Lee W, Cahill P, Honan T, Sparrow T, Weiand M, et al. Highresolution description of antibody heavy-chain repertoires in humans. PLoS One (2011) 6(8):e22365. doi:10.1371/journal.pone.0022365

13. UniProt Consortium. UniProt: a hub for protein information. Nucleic Acids Res (2015) 43(Database issue):D204-12. doi:10.1093/nar/gku989

14. Wattam AR, Abraham D, Dalay O, Disz TL, Driscoll T, Gabbard JL, et al. PATRIC, the bacterial bioinformatics database and analysis resource. Nucleic Acids Res (2014) 42(Database issue):D581-91. doi:10.1093/nar/gkt1099

15. Lilliefors HW. On the Kolmogorov-Smirnov test for normality with mean and variance unknown. J Am Stat Assoc (1967) 62:399-402. doi:10.1080/0162145 9.1967.10482916

16. Petrova GV, Gorski J. Cross-reactive responses to modified M1(5)(8)-(6)(6) peptides by CD8(+) $\mathrm{T}$ cells that use noncanonical BV genes can describe unknown repertoires. Eur J Immunol (2012) 42(11):3001-8. doi:10.1002/ eji.201242596

17. Nelson RW, Beisang D, Tubo NJ, Dileepan T, Wiesner DL, Nielsen K, et al. $\mathrm{T}$ cell receptor cross-reactivity between similar foreign and self peptides influences naive cell population size and autoimmunity. Immunity (2015) 42(1):95-107. doi:10.1016/j.immuni.2014.12.022

18. Bremel RD, Homan EJ. An integrated approach to epitope analysis II: a system for proteomic-scale prediction of immunological characteristics. Immunome Res (2010) 6(1):8. doi:10.1186/1745-7580-6-8

19. Moise L, Gutierrez AH, Bailey-Kellogg C, Terry F, Leng Q, Abdel Hady KM, et al. The two-faced T cell epitope: examining the host-microbe interface with JanusMatrix. Hum Vaccin Immunother (2013) 9(7):1577-86. doi:10.4161/ hv. 24615

20. Birnbaum ME, Mendoza JL, Sethi DK, Dong S, Glanville J, Dobbins J, et al. Deconstructing the peptide-MHC specificity of T cell recognition. Cell (2014) 157(5):1073-87. doi:10.1016/j.cell.2014.03.047

21. Gett AV, Sallusto F, Lanzavecchia A, Geginat J. T cell fitness determined by signal strength. Nat Immunol (2003) 4(4):355-60. doi:10.1038/ni908

22. Jenkins MK, Moon JJ. The role of naive $\mathrm{T}$ cell precursor frequency and recruitment in dictating immune response magnitude. JImmunol (2012) 188(9):4135-40. doi:10.4049/jimmunol.1102661

23. Josefowicz SZ, Lu LF, Rudensky AY. Regulatory T cells: mechanisms of differentiation and function. Annu Rev Immunol (2012) 30:531-64. doi:10.1146/ annurev.immunol.25.022106.141623

24. Ohkura N, Hamaguchi M, Morikawa H, Sugimura K, Tanaka A, Ito Y, et al. $\mathrm{T}$ cell receptor stimulation-induced epigenetic changes and Foxp3 expression are independent and complementary events required for Treg cell development. Immunity (2012) 37(5):785-99. doi:10.1016/j.immuni.2012.09.010

25. Vahl JC, Drees C, Heger K, Heink S, Fischer JC, Nedjic J, et al. Continuous T cell receptor signals maintain a functional regulatory $\mathrm{T}$ cell pool. Immunity (2014) 41(5):722-36. doi:10.1016/j.immuni.2014.10.012

26. Liu B, Chen W, Evavold BD, Zhu C. Accumulation of dynamic catch bonds between TCR and agonist peptide-MHC triggers T cell signaling. Cell (2014) 157(2):357-68. doi:10.1016/j.cell.2014.02.053

27. Sakaguchi S, Sakaguchi N, Shimizu J, Yamazaki S, Sakihama T, Itoh M, et al. Immunologic tolerance maintained by $\mathrm{CD} 25+\mathrm{CD} 4+$ regulatory $\mathrm{T}$ cells: their common role in controlling autoimmunity, tumor immunity, and transplantation tolerance. Immunol Rev (2001) 182:18-32. doi:10.1034/j.1600-065X.2001.1820102.x

28. Hofer T, Krichevsky O, Altan-Bonnet G. Competition for IL-2 between regulatory and effector $\mathrm{T}$ cells to chisel immune responses. Front Immunol (2012) 3:268. doi:10.3389/fimmu.2012.00268

29. Smigiel KS, Srivastava S, Stolley JM, Campbell DJ. Regulatory T-cell homeostasis: steady-state maintenance and modulation during inflammation. Immunol Rev (2014) 259(1):40-59. doi:10.1111/imr.12170

30. Leignadier J, Labrecque N. Epitope density influences CD8+ memory T cell differentiation. PLoS One (2010) 5(10):e13740. doi:10.1371/journal. pone. 0013740

31. Almeida AR, Amado IF, Reynolds J, Berges J, Lythe G, Molina-Paris C, et al. Quorum-sensing in CD4(+) T cell homeostasis: a hypothesis and a model. Front Immunol (2012) 3:125. doi:10.3389/fimmu.2012.00125
32. Petrova G, Ferrante A, Gorski J. Cross-reactivity of T cells and its role in the immune system. Crit Rev Immunol (2012) 32(4):349-72. doi:10.1615/ CritRevImmunol.v32.i4.50

33. Painter CA, Stern LJ. Conformational variation in structures of classical and non-classical MHCII proteins and functional implications. Immunol Rev (2012) 250(1):144-57. doi:10.1111/imr.12003

34. Bodelon G, Palomino C, Fernandez LA. Immunoglobulin domains in Escherichia coli and other enterobacteria: from pathogenesis to applications in antibody technologies. FEMS Microbiol Rev (2013) 37(2):204-50. doi:10.1111/j.1574-6976.2012.00347.x

35. Belkaid Y, Hand TW. Role of the microbiota in immunity and inflammation. Cell (2014) 157(1):121-41. doi:10.1016/j.cell.2014.03.011

36. Attridge $\mathrm{K}$, Walker LS. Homeostasis and function of regulatory $\mathrm{T}$ cells (Tregs) in vivo: lessons from TCR-transgenic Tregs. Immunol Rev (2014) 259(1):23-39. doi:10.1111/imr.12165

37. Caton AJ, Kropf E, Simons DM, Aitken M, Weissler KA, Jordan MS. Strength of TCR signal from self-peptide modulates autoreactive thymocyte deletion and Foxp3(+) Treg-cell formation. Eur JImmunol (2014) 44(3):785-93. doi:10.1002/eji.201343767

38. Bluestone JA, Mackay CR, O'Shea JJ, Stockinger B. The functional plasticity of T cell subsets. Nat Rev Immunol (2009) 9(11):811-6. doi:10.1038/ nri2654

39. Sawant DV, Vignali DA. Once a Treg, always a Treg? Immunol Rev (2014) 259(1):173-91. doi:10.1111/imr.12173

40. Hsieh CS, Zheng Y, Liang Y, Fontenot JD, Rudensky AY. An intersection between the self-reactive regulatory and nonregulatory $\mathrm{T}$ cell receptor repertoires. Nat Immunol (2006) 7(4):401-10. doi:10.1038/ni1318

41. Barnes MJ, Griseri T, Johnson AM, Young W, Powrie F, Izcue A. CTLA-4 promotes Foxp3 induction and regulatory $\mathrm{T}$ cell accumulation in the intestinal lamina propria. Mucosal Immunol (2013) 6(2):324-34. doi:10.1038/ mi.2012.75

42. Sathaliyawala T, Kubota M, Yudanin N, Turner D, Camp P, Thome JJ, et al. Distribution and compartmentalization of human circulating and tissue-resident memory T cell subsets. Immunity (2013) 38(1):187-97. doi:10.1016/j. immuni.2012.09.020

43. Fulcher DA, Basten A. B cell life span: a review. Immunol Cell Biol (1997) 75(5):446-55. doi:10.1038/icb.1997.69

44. Bonilla FA. Pharmacokinetics of immunoglobulin administered via intravenous or subcutaneous routes. Immunol Allergy Clin North Am (2008) 28(4):803-19. doi:10.1016/j.iac.2008.06.006

45. Dati F, Schumann G, Thomas L, Aguzzi F, Baudner S, Bienvenu J, et al. Consensus of a group of professional societies and diagnostic companies on guidelines for interim reference ranges for 14 proteins in serum based on the standardization against the IFCC/BCR/CAP Reference Material (CRM 470). International Federation of Clinical Chemistry. Community Bureau of Reference of the Commission of the European Communities. College of American Pathologists. Eur J Clin Chem Clin Biochem (1996) 34(6):517-20.

46. Gonzalez-Quintela A, Alende R, Gude F, Campos J, Rey J, Meijide LM, et al. Serum levels of immunoglobulins (IgG, $\operatorname{IgA}$, IgM) in a general adult population and their relationship with alcohol consumption, smoking and common metabolic abnormalities. Clin Exp Immunol (2008) 151(1):42-50. doi:10.1111/j.1365-2249.2007.03545.x

47. Su LF, Kidd BA, Han A, Kotzin JJ, Davis MM. Virus-specific CD4(+) memory-phenotype $\mathrm{T}$ cells are abundant in unexposed adults. Immunity (2013) 38(2):373-83. doi:10.1016/j.immuni.2012.10.021

48. Neller MA, Ladell K, McLaren JE, Matthews KK, Gostick E, Pentier JM, et al. Naive CD8 T-cell precursors display structured TCR repertoires and composite antigen-driven selection dynamics. Immunol Cell Biol (2015) 93(7):625-33. doi:10.1038/icb.2015.17

49. Boer MC, Joosten SA, Ottenhoff TH. Regulatory T-cells at the interface between human host and pathogens in infectious diseases and vaccination. Front Immunol (2015) 6:217. doi:10.3389/fimmu.2015.00217

50. Boer MC, van Meijgaarden KE, Joosten SA, Ottenhoff TH. CD8+ regulatory $\mathrm{T}$ cells, and not CD4+ T cells, dominate suppressive phenotype and function after in vitro live Mycobacterium bovis-BCG activation of human cells. PLoS One (2014) 9(4):e94192. doi:10.1371/journal.pone.0094192 
51. Sewell AK. Why must T cells be cross-reactive? Nat Rev Immunol (2012) 12(9):669-77. doi:10.1038/nri3279

52. Wooldridge L, Ekeruche-Makinde J, van den Berg HA, Skowera A, Miles JJ, Tan MP, et al. A single autoimmune $\mathrm{T}$ cell receptor recognizes more than a million different peptides. J Biol Chem (2012) 287(2):1168-77. doi:10.1074/ jbc.M111.289488

53. Wucherpfennig KW, Allen PM, Celada F, Cohen IR, De Boer R, Garcia KC, et al. Polyspecificity of T cell and B cell receptor recognition. Semin Immunol (2007) 19(4):216-24. doi:10.1016/j.smim.2007.02.012
Conflict of Interest Statement: Both authors are employees and equity holders in ioGenetics LLC, the parent company of EigenBio LLC.

Copyright (๑) 2015 Bremel and Homan. This is an open-access article distributed under the terms of the Creative Commons Attribution License (CC BY). The use, distribution or reproduction in other forums is permitted, provided the original author(s) or licensor are credited and that the original publication in this journal is cited, in accordance with accepted academic practice. No use, distribution or reproduction is permitted which does not comply with these terms. 